\title{
UK Renal Registry 15th Annual Report: Chapter 2 UK RRT Prevalence in 2011: National and Centre-Specific Analyses
}

\author{
Catriona Shaw ${ }^{\mathrm{a}}$, Rishi Pruthi ${ }^{\mathrm{a}}$, David Pitcher $^{\mathrm{a}}$, Damian Fogarty ${ }^{\mathrm{ab}}$ \\ ${ }^{\mathrm{a} U K}$ Renal Registry, Bristol, UK; ${ }^{\mathrm{b}}$ Queens University, Belfast, UK
}

\begin{abstract}
Key Words
Chronic kidney disease - Comorbidity - Diabetes - Dialysis . End stage renal disease - Established renal failure - Ethnicity - Haemodialysis - Peritoneal dialysis - Prevalence - Primary Care Trust - Renal replacement therapy - Transplantation . Treatment modality
\end{abstract}

\begin{abstract}
Introduction: Introduction: This chapter describes the characteristics of adult patients on renal replacement therapy (RRT) in the UK in 2011. The prevalence rates per million population (pmp) were calculated for Primary Care Trusts in England, Health and Social Care areas in Northern Ireland, Local Health Boards in Wales and Health Boards (HB) in Scotland (PCT/HB areas). Methods: Data were electronically collected from all 71 renal centres within the UK. A series of cross-sectional and longitudinal analyses were performed to describe the demographics of prevalent RRT patients in 2011 at centre and national level. Age and gender standardised ratios for prevalence rates in PCT/ HBs were calculated. Results: There were 53,207 adult patients receiving RRT in the UK on 31st December 2011. The UK adult prevalence of RRT was $842 \mathrm{pmp}$. This represented an annual increase in prevalent numbers of approximately $4 \%$, although there was variation between
\end{abstract}

centres and PCT/HB areas. The growth rate from 2010 to 2011 for prevalent patients by treatment modality in the UK was an increase of $1.7 \%$ for haemodialysis (HD), a fall of $2.2 \%$ for peritoneal dialysis (PD) and an increase of $4.7 \%$ with a functioning transplant. There has been a slow but steady decline in the proportion of dialysis patients receiving PD since 2000. In contrast, the number of patients receiving home HD has increased $16 \%$ since 2010 . Median RRT vintage for patients on HD was 3.3 years, PD 1.8 years and for those patients with a transplant, 10.3 years. The median age of prevalent patients was 58.2 years (HD 66.5 years, PD 62.7 years, transplant 51.7 years) compared to 55 years in 2000. For all ages the prevalence rate in men exceeded that in women, peaking in age group 75-79 years at $2,918 \mathrm{pmp}$ in males. For females the peak was in age group 65-69 years at 1,460 pmp. The most common recorded renal diagnosis was glomerulonephritis (biopsy proven/not biopsy proven) (19\%), followed by uncertain (18\%). Transplantation was the most common treatment modality (48.6\%), HD in $43.9 \%$ and PD $7.6 \%$. However, HD was increasingly common with increasing age and transplantation less common. Conclusions: The HD and transplant population continued to expand whilst the PD population contracted. There were national, regional and dialysis centre level variations in prevalence rates. Prevalent patients were on average three years older than 10 years ago. This has implications for service planning and ensuring equity of care for RRT patients.
Catriona Shaw

UK Renal Registry, Southmead Hospital, Southmead Road, Bristol, BS10 5NB, UK

Email: renalregistry@renalregistry.nhs.uk 


\section{Introduction}

This chapter presents data on all adult patients on RRT in the UK at the end of 2011. The UK Renal Registry (UKRR) received data returns for 2011 from all five renal centres in Wales, all five in Northern Ireland and all 52 in England. Data from all nine centres in Scotland were obtained from the Scottish Renal Registry. Data on children and young adults can be found in chapter 4 .

These analyses of prevalent RRT patients are performed annually to aid clinicians and policy makers in planning future RRT requirements in the UK. It is important to understand national, regional and centre level variation in numbers of prevalent patients as part of the planning process. In addition, knowledge about variation in case mix is also reported to improve understanding of where resources should be focussed to improve equity of provision of RRT in the UK.

The term established renal failure (ERF) used within this chapter is synonymous with the terms end stage renal failure and end stage renal disease, which are in more widespread international usage. Patients have disliked the term 'end stage' which reflects the inevitable outcome of this disease.

\section{Methods}

These analyses relate to the prevalent RRT cohort in the UK in 2011. The cohort was defined as all adult patients receiving RRT on the UKRR database on 31st December 2011. Population estimates were obtained from the UK Office of National Statistics (ONS) [1].

The number of adult prevalent RRT patients was calculated for the UK as a whole and for each UK country, using UKRR data from all renal centres. Crude prevalence rates were calculated per million population ( $\mathrm{pmp}$ ) and standardised prevalence ratios were calculated as detailed in appendix D: Methodology used for Analyses (www.renalreg.com) for Primary Care Trusts (PCT) in England, Health \& Social Care Areas in Northern Ireland, Local Health Boards in Wales and Health Boards in Scotland. These areas will be referred to in this report as 'PCT/HBs'. Briefly, data from all areas were used to calculate overall age and gender specific prevalence rates. The age and gender breakdown of the population in each PCT/HB were obtained from the mid-2010 population estimate based on 2001 Census data from the ONS [1]. The population breakdown and the overall prevalence rates were used to calculate the expected age and gender specific prevalence numbers for each $\mathrm{PCT} / \mathrm{HB}$. The age and gender standardised prevalence ratio was the observed prevalence number divided by the expected prevalence number. A ratio below 1 indicated that the observed rate was less than expected given the area's population structure. This was statistically significant at the 5\% level if the upper confidence limit was less than 1. Analyses were done for each of the last 6 years and as the prevalent numbers for one year can be small for smaller areas, a combined years' analysis was also done. To enable assessment of whether a centre was an outlier in this regard, funnel plots for smaller and larger populations have been included (appendix D: figures D3, D4) which show the 95\% confidence intervals around the national average prevalence. The proportion of nonWhites in each PCT/HB was obtained from the ONS [1].

This year there are a total of 71 renal centres, whereas in previous reports there have been 72 . This is due to a merging of the Derry and Tyrone renal centres in Northern Ireland. The prevalence rate per million population for each centre was calculated using a derived catchment population. This was calculated from the postcode of each prevalent patient in 2007 and the population within that postcode assigned to the renal centre where that patient was treated. For a full description of the methodology used to estimate the catchment populations see appendix E: Methodology for Estimating Catchment Populations Analyses (www.renalreg.com). In brief, the patient postcode for each prevalent dialysis patient in 2007 was used to create a series of overlapping areas corresponding to each renal centre. These small areas were then assigned to a Census Area Statistics ward using geographical information system technology and the population in each area assigned to its respective renal centre. These estimates will not be accurate for new centres and centres with changes in catchment populations since 2007 (e.g. Bristol, Cambridge and Ipswich, which have lost catchment population since 2007 and Dorset which gained catchment population); in addition the analysis used dialysis patients only and transplant patients may come from a different catchment population. Estimation of catchment populations therefore remains an inexact science and these figures should be regarded as indicative only. This methodology was used for England only. Estimates of the catchment populations in Wales and Northern Ireland were supplied by personal communication from Dr K Donovan, Dr A Williams and Dr D Fogarty.

Throughout this chapter, haemodialysis refers to all modes of HD treatment, including haemodiafiltration (HDF). Several centres reported significant numbers of patients on HDF, but other centres did not differentiate this treatment type in their UKRR returns. Where joint care of renal transplant recipients between the referring centre and the transplant centre occurred, the patient was allocated to the centre which saw the patient most frequently, usually the referring centre. Thus the number of patients allocated to a transplant centre is often lower than that recorded by the centre itself and as a converse pre-emptively transplanted patients are sometimes allocated to the transplanting centre rather than the referring centre if no transfer out code had been sent through. Queries and updated information are welcomed by the UKRR at any point during the year if this has occurred.

Prevalent patients on RRT in 2011 were examined by time on RRT, age group, gender, ethnic origin, primary renal disease, presence of diabetes and treatment modality (2009 Report appendix $\mathrm{H}$ : Coding (www.renalreg.com)). In this year's analysis of prevalence, only adult patients on RRT contributed to the numerator. In previous years, children had been included in the numerator also. Data on the paediatric population is presented in Chapter 4. Some centres electronically upload ethnicity coding to their renal information technology (IT) system from the hospital 
Table 2.1. Prevalence of adult RRT in the UK on $31 / 12 / 2011$

\begin{tabular}{|c|c|c|c|c|c|}
\hline & England & $\mathrm{N}$ Ireland & Scotland & Wales & UK \\
\hline All UK centres & 44,665 & 1,510 & 4,324 & 2,708 & 53,207 \\
\hline Total estimated population, mid-2010 (millions) ${ }^{*}$ & 53.0 & 1.8 & 5.3 & 3.1 & 63.2 \\
\hline Prevalence rate PD (pmp) & 62 & 43 & 47 & 72 & 61 \\
\hline Prevalence rate dialysis (pmp) & 427 & 443 & 402 & 433 & 426 \\
\hline Prevalence rate transplant (pmp) & 415 & 390 & 415 & 451 & 416 \\
\hline
\end{tabular}

* estimates from ONS web site

$\mathrm{pmp}=$ per million population

Patient Administration System (PAS). Ethnicity coding in these PAS systems is based on self-reported ethnicity and uses a different coding system to those centres not linked to PAS [2]. For the remaining centres, ethnicity coding is performed by clinical staff and recorded directly into the renal IT system (using a variety of coding systems). For all these analyses, data on ethnic origin were grouped into Whites, South Asians, Blacks, Chinese and Others as described in appendix $\mathrm{H}$ : Coding (www.renalreg. com). This year, individuals with a primary renal diagnosis (PRD) 'glomerulonephritis biopsy unproven' were grouped within the 'glomerulonephritis' PRD group, rather than within 'uncertain' (as has been the case in previous reports) to reflect better coding and bringing the registry in line with coding methodology adopted in other renal registries. Time on RRT was defined as median time on treatment and was calculated from the most recent start date. Patients without an accurate start date were excluded from this calculation. Analyses were done for the UK as a whole, by UK country, at centre level and split by treatment modality when appropriate.

Chi-squared test, Fisher's exact test, linear regression and Kruskal Wallis tests were used as appropriate to test for significant differences between groups. The data were analysed using SAS 9.3.

\section{Results}

\section{Prevalent patient numbers and changes in prevalence}

The number of patients for each country (table 2.1) was calculated by adding the patient numbers in each renal centre and these differ marginally from those quoted elsewhere when patients are allocated to geographical areas by their individual postcodes, as some centres treat patients across national boundaries.

There were 53,207 adult patients receiving RRT in the UK at the end of 2011, giving an adult UK population prevalence of $842 \mathrm{pmp}$ (table 2.1) compared with $832 \mathrm{pmp}$ in 2010 [3]. Prevalence rates increased in all of the UK countries in 2011 except Scotland were there was a small decline from $829 \mathrm{pmp}$ in 2010 to $817 \mathrm{pmp}$ in 2011. PD prevalence increased in Northern Ireland but decreased in the other three countries compared with 2010. The overall decline in PD prevalence in the UK has been a consistent pattern observed since 1997. Once more, the prevalence of transplanted patients increased in the UK. Northern Ireland had a higher RRT prevalence rate for patients aged 65 and older compared with the other UK countries (figure 2.1). In the UK, the RRT prevalence rate in patients aged $80-84$ continued to rise over time from 1,220 per million age related population (pmarp) in 2006 to 1,824 pmarp in 2011 and in patients aged $>85$ years from 524 pmarp in 2006 to 952 pmarp in 2011. It is likely that this ageing of the prevalent population was due to an increasing numbers of older patients starting RRT, although improving patient survival will also contribute.

\section{Prevalent patients by RRT centre}

The number of prevalent patients in each renal centre and the distribution of their treatment modalities varied widely (table 2.2). Many factors including geography, local population density, age distribution, ethnic

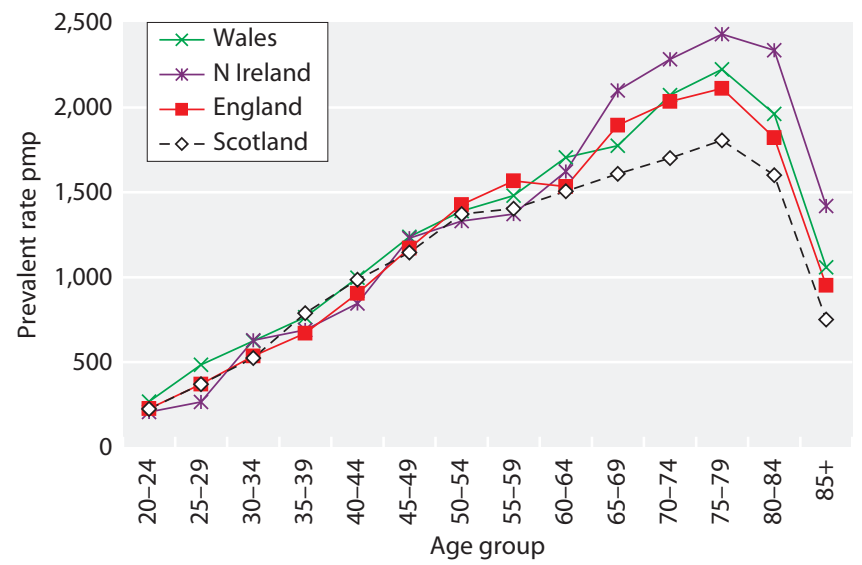

Fig. 2.1. Prevalence rates per million population by age group and UK country on $31 / 12 / 2011$ 
Table 2.2. Number of prevalent RRT patients by treatment modality and centre on 31/12/2011

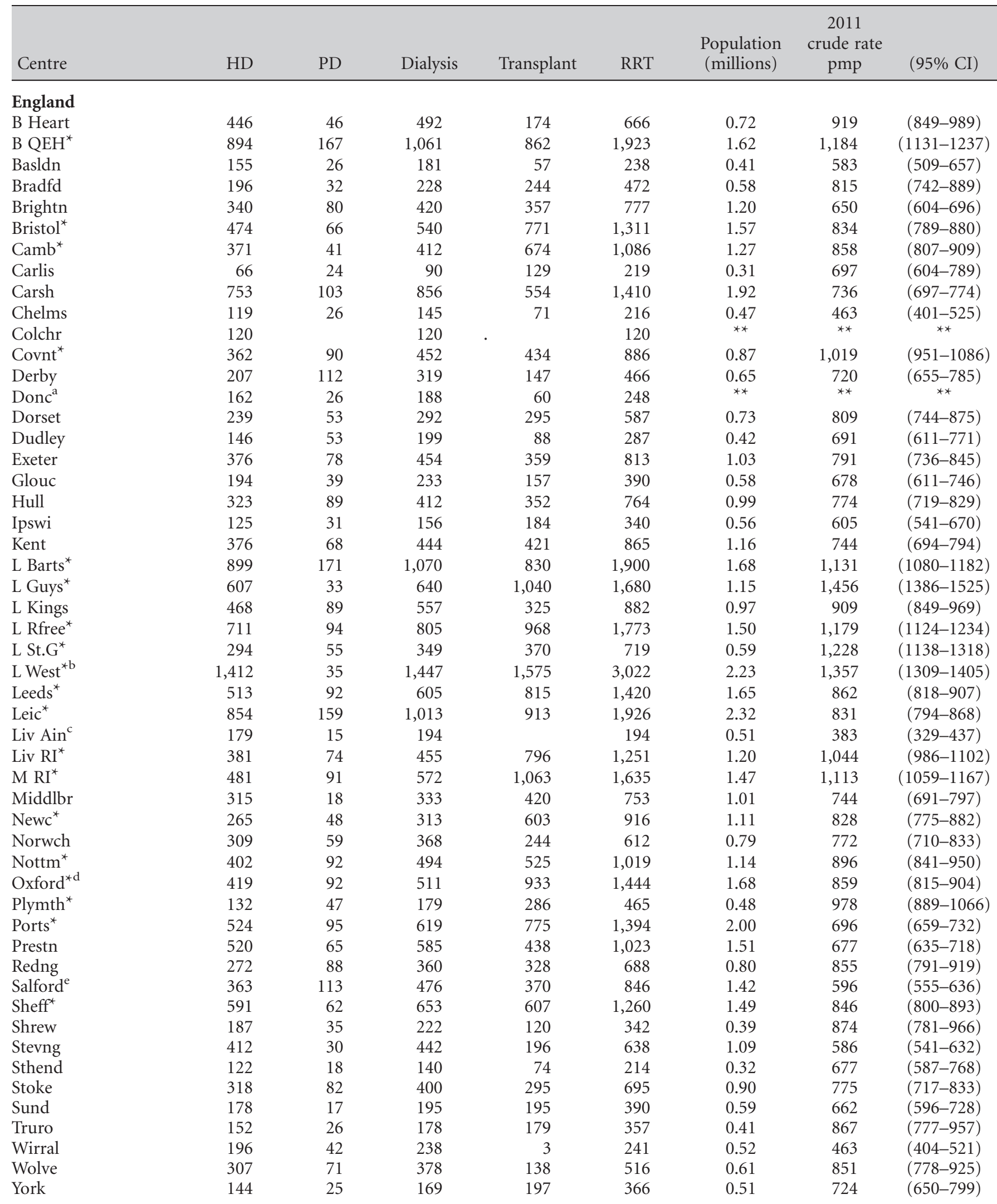


Table 2.2. Continued

\begin{tabular}{|c|c|c|c|c|c|c|c|c|}
\hline Centre & $\mathrm{HD}$ & PD & Dialysis & Transplant & RRT & $\begin{array}{c}\text { Population } \\
\text { (millions) }\end{array}$ & $\begin{array}{c}2011 \\
\text { crude rate } \\
\text { pmp }\end{array}$ & $(95 \% \mathrm{CI})$ \\
\hline \multicolumn{9}{|c|}{ Northern Ireland } \\
\hline Antrim & 132 & 14 & 146 & 78 & 224 & 0.30 & 747 & $(649-844)$ \\
\hline Belfast $^{*}$ & 228 & 30 & 258 & 428 & 686 & 0.55 & 1,241 & $(1148-1333)$ \\
\hline Newry & 111 & 12 & 123 & 68 & 191 & 0.28 & 675 & $(579-771)$ \\
\hline Ulster & 105 & 3 & 108 & 29 & 137 & 0.30 & 457 & $(380-533)$ \\
\hline West NI ${ }^{\mathrm{f}}$ & 149 & 19 & 168 & 104 & 272 & 0.35 & 771 & $(679-862)$ \\
\hline \multicolumn{9}{|l|}{ Scotland } \\
\hline Abrdn & 214 & 23 & 237 & 242 & 479 & 0.60 & 801 & $(729-873)$ \\
\hline Airdrie & 173 & 10 & 183 & 161 & 344 & 0.56 & 611 & $(546-675)$ \\
\hline D \& Gall & 49 & 14 & 63 & 59 & 122 & 0.15 & 824 & $(678-970)$ \\
\hline Dundee & 183 & 22 & 205 & 195 & 400 & 0.41 & 986 & $(889-1083)$ \\
\hline Dunfn & 146 & 28 & 174 & 104 & 278 & 0.37 & 757 & $(668-846)$ \\
\hline Edinb $^{*}$ & 261 & 40 & 301 & 399 & 700 & 0.96 & 728 & $(674-782)$ \\
\hline Glasgw $^{\star}$ & 622 & 49 & 671 & 806 & 1,477 & 1.51 & 981 & $(931-1031)$ \\
\hline Inverns & 83 & 18 & 101 & 123 & 224 & 0.34 & 663 & $(576-749)$ \\
\hline Klmarnk & 147 & 45 & 192 & 108 & 300 & 0.37 & 818 & $(725-910)$ \\
\hline \multicolumn{9}{|l|}{ Wales } \\
\hline Bangor & 88 & 21 & 109 & & 109 & 0.25 & 436 & $(354-518)$ \\
\hline Cardff $^{*}$ & 495 & 102 & 597 & 939 & 1,536 & 1.45 & 1,059 & $(1006-1112)$ \\
\hline Clwyd & 76 & 20 & 96 & 71 & 167 & 0.20 & 835 & $(708-962)$ \\
\hline Swanse & 358 & 58 & 416 & 243 & 659 & 0.80 & 824 & $(761-887)$ \\
\hline Wrexm & 88 & 20 & 108 & 129 & 237 & 0.30 & 790 & $(689-891)$ \\
\hline England & 19,371 & 3,283 & 22,654 & 22,011 & 44,665 & & & \\
\hline N Ireland & 725 & 78 & 803 & 707 & 1,510 & & & \\
\hline Scotland ${ }^{g}$ & 1,878 & 249 & 2,127 & 2,197 & 4,324 & & & \\
\hline Wales & 1,105 & 221 & 1,326 & 1,382 & 2,708 & & & \\
\hline UK & 23,079 & 3,831 & 26,910 & 26,297 & 53,207 & & & \\
\hline
\end{tabular}

Blank cells indicate no patients on that treatment attending that unit when data was collected

Centres prefixed 'L' are London centres

The numbers of patients calculated for each country quoted above differ marginally from those quoted elsewhere when patients are allocated to areas by their individual post codes, as some centres treat patients from across national boundaries

${ }^{*}$ Transplant centres

${ }^{*}$ Doncaster and Colchester were not established main renal centres when the catchment population work was undertaken

${ }^{a}$ Doncaster previously a satellite of Sheffield

${ }^{\mathrm{b}}$ Hammersmith and Charing Cross amalgamated with St Mary's

${ }^{\mathrm{c}}$ Liv Ain catchment population updated after correspondence with the centre

${ }^{\mathrm{d}}$ Oxford transferred Northamptonshire local authority to Leicester

e Salford previously named Manchester Hope

${ }^{\mathrm{f}}$ West NI is the amalgamation of Derry and Tyrone

${ }^{\mathrm{g}}$ Scotland catchment populations correct as at 30 June 2011

composition, prevalence of diseases predisposing to kidney disease and the social deprivation index of that population may contribute to this.

\section{Changes in prevalence}

Overall growth in the prevalent UK RRT population from 2010 to 2011 was $4.3 \%$ (table 2.3), an annual growth rate which has been fairly consistent over the last 10-15 years (figure 2.2). Most of the growth in the prevalent RRT population was due to a continued increase in the size of the prevalent RRT population in England, Wales and Northern Ireland, with slower growth in the prevalent RRT population in Scotland. The most substantial changes in relative size of the prevalent population were in Northern Ireland, which increased from a $1.0 \%$ change in the size of the prevalent population in 2009-2010 to $4.4 \%$ in 2010-2011, and in Scotland, which saw a decline from an increase in the prevalent RRT population of $3.5 \%$ in 2009-2010 to $1.2 \%$ in the most recent analysis. 
Table 2.3. Number of prevalent patients on RRT by centre at year end 2007-2011*

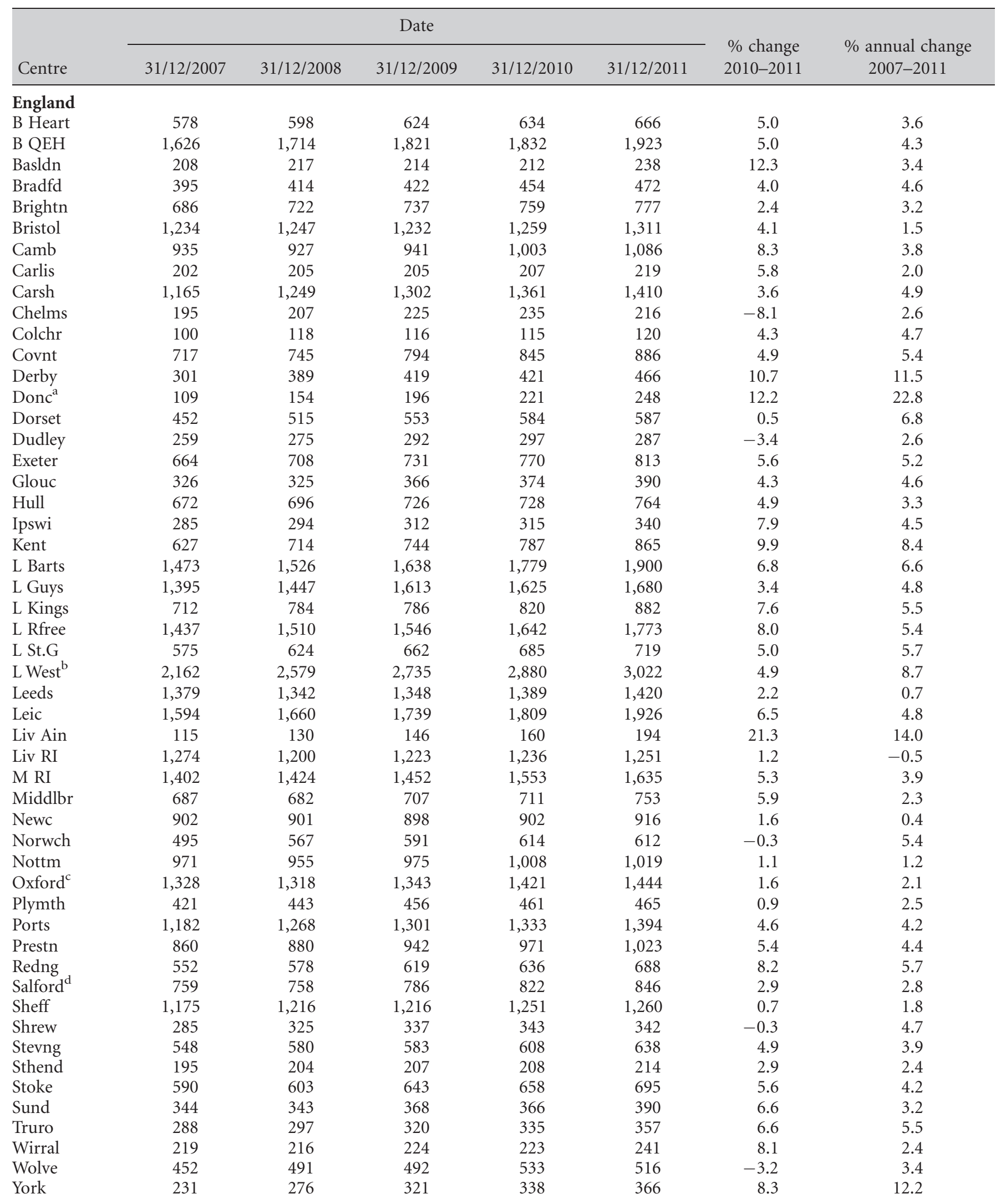


Table 2.3. Continued

\begin{tabular}{|c|c|c|c|c|c|c|c|}
\hline \multirow[b]{2}{*}{ Centre } & \multicolumn{5}{|c|}{ Date } & \multirow{2}{*}{$\begin{array}{l}\text { \% change } \\
2010-2011\end{array}$} & \multirow{2}{*}{$\begin{array}{c}\% \text { annual change } \\
\text { 2007-2011 }\end{array}$} \\
\hline & $31 / 12 / 2007$ & $31 / 12 / 2008$ & $31 / 12 / 2009$ & $31 / 12 / 2010$ & $31 / 12 / 2011$ & & \\
\hline \multicolumn{8}{|l|}{ N Ireland } \\
\hline Antrim & 200 & 220 & 215 & 214 & 224 & 4.7 & 2.9 \\
\hline Belfast & 748 & 726 & 680 & 680 & 686 & 0.9 & -2.1 \\
\hline Newry & 148 & 163 & 171 & 179 & 191 & 6.7 & 6.6 \\
\hline Ulster & 90 & 97 & 114 & 115 & 137 & 19.1 & 11.1 \\
\hline West $\mathrm{NI}^{\mathrm{e}}$ & 216 & 236 & 258 & 258 & 272 & 5.4 & 5.9 \\
\hline \multicolumn{8}{|l|}{ Scotland } \\
\hline Abrdn & 452 & 456 & 452 & 463 & 479 & 3.5 & 1.5 \\
\hline Airdrie & 231 & 245 & 310 & 309 & 344 & 11.3 & 10.5 \\
\hline D \& Gall & 77 & 113 & 118 & 115 & 122 & 6.1 & 12.2 \\
\hline Dundee & 376 & 370 & 395 & 382 & 400 & 4.7 & 1.6 \\
\hline Dunfn & 220 & 220 & 241 & 257 & 278 & 8.2 & 6.0 \\
\hline Edinb & 720 & 695 & 721 & 731 & 700 & -4.2 & -0.7 \\
\hline Glasgw & 1,605 & 1,568 & 1,469 & 1,505 & 1,477 & -1.9 & -2.1 \\
\hline Inverns & 214 & 212 & 228 & 230 & 224 & -2.6 & 1.1 \\
\hline Klmarnk & 214 & 263 & 273 & 282 & 300 & 6.4 & 8.8 \\
\hline \multicolumn{8}{|l|}{ Wales } \\
\hline Bangor & 98 & 112 & 110 & 113 & 109 & -3.5 & 2.7 \\
\hline Cardff & 1,438 & 1,375 & 1,428 & 1,481 & 1,536 & 3.7 & 1.7 \\
\hline Clwyd & 155 & 146 & 144 & 136 & 167 & 22.8 & 1.9 \\
\hline Swanse & 545 & 602 & 598 & 630 & 659 & 4.6 & 4.9 \\
\hline Wrexm & 213 & 223 & 219 & 221 & 237 & 7.2 & 2.7 \\
\hline England & 37,738 & 39,560 & 41,189 & 42,733 & 44,665 & 4.5 & 4.3 \\
\hline N Ireland & 1,402 & 1,442 & 1,438 & 1,446 & 1,510 & 4.4 & 1.9 \\
\hline Scotland & 4,109 & 4,142 & 4,207 & 4,274 & 4,324 & 1.2 & 1.3 \\
\hline Wales & 2,449 & 2,458 & 2,499 & 2,581 & 2,708 & 4.9 & 2.5 \\
\hline UK & 45,698 & 47,602 & 49,333 & 51,034 & 53,207 & 4.3 & 3.9 \\
\hline
\end{tabular}

${ }^{a}$ Doncaster previously a satellite of Sheffield

${ }^{\mathrm{b}}$ Hammersmith and Charing Cross amalgamated with St Mary's

${ }^{c}$ Oxford transferred Northamptonshire local authority to Leicester

${ }^{\mathrm{d}}$ Salford previously named Manchester Hope

${ }^{\mathrm{e}}$ West NI is the amalgamation of Derry and Tyrone

${ }^{*}$ After confirmation of the numbers of patients with renal centres several inaccuracies were identified. In Kent 16 additional transplant patients and in York 27 additional transplant patients were identified. In Leeds the transplant population had been overestimated by 21 . In Clwyd an additional $13 \mathrm{HD}$ patients, $12 \mathrm{PD}$ patients and 6 transplant patients were identified. These changes have been incorporated into tables $2.1,2.2$ and 2.3 but not any other analyses

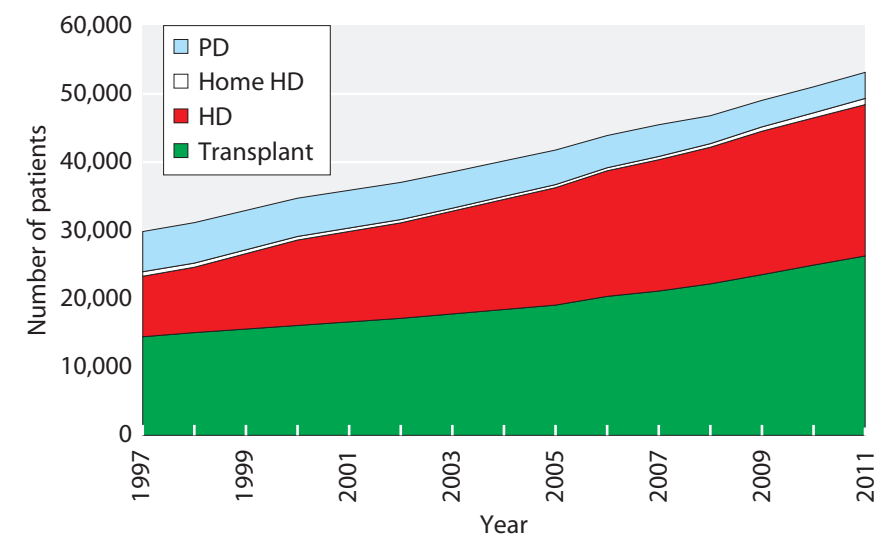

Fig. 2.2. Growth in prevalent patients by treatment modality at the end of each year 1997-2011
Across the different RRT modalities (HD, PD and transplant) there was heterogeneity in the prevalent growth per million population (pmp), as shown in table 2.4. From 2010 to 2011 , there was a $1.7 \%$ growth in prevalent HD patients, a $4.7 \%$ growth in those with a functioning transplant and a $2.2 \%$ decline in patients on PD. Between 2006 and 2011 there was an average annual $3.3 \%$ pmp growth in $\mathrm{HD}, 4.9 \%$ pmp fall in $\mathrm{PD}$, and $4.4 \%$ pmp growth in prevalent transplant patients in the UK (table 2.4). In the same period there was a $103 \%$ increase in the use of home haemodialysis (445 patients to 905 patients).

There were large variations in RRT prevalence between centres as well as countries. For example, from 
Table 2.4. Change in RRT prevalence rates pmp 2006-2011 by modality

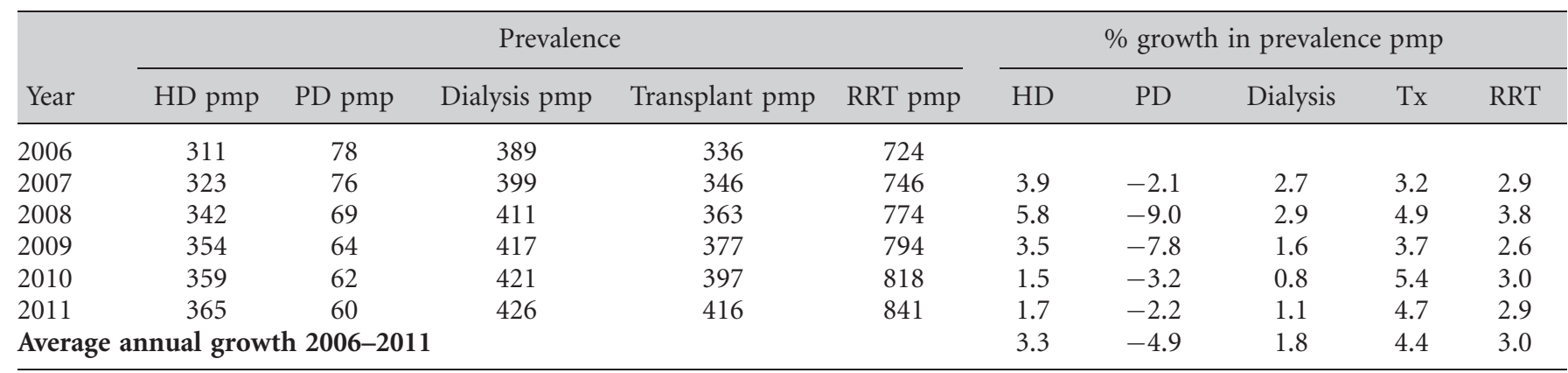

${ }^{\star}$ Differences in the figures for dialysis and RRT prevalence and the sum of the separate modalities are due to rounding

pmp - per million population

2010 to 2011 the number of prevalent patients on RRT at Liverpool Aintree increased by $21.3 \%$, whilst in Chelmsford the number decreased by $8.1 \%$ (table 2.3). These changes could be related to re-allocation of patients from and to other local renal centres. Centre prevalence rates showed marked variation; from $457 \mathrm{pmp}$ in Ulster to $1,456 \mathrm{pmp}$ at London Guy's (table 2.2). The longterm (1997-2010) UK prevalence pattern by treatment modality is shown in figure 2.2. The steady growth in transplant numbers was maintained in 2011. The increase in transplant prevalence and the increase in haemodialysis patient numbers has been associated with a slow contraction in home-based therapies, particularly $\mathrm{PD}$, in more recent years.

Prevalence of RRT in Primary Care Trusts (PCT) in England, Health and Social Care Areas in Northern Ireland (HB), Local Health Boards in Wales (HB) and Health Boards in Scotland (HB)

The need for RRT depends on many factors such as predisposing conditions but also on social and demographic factors such as age, gender, social deprivation and ethnicity. Hence, comparison of crude prevalence rates by geographical area can be misleading. This section, as in previous reports, uses age and gender standardisation to compare RRT prevalence rates. The ethnic minority profile is also provided to help understand the differences in standardised prevalence ratios (SPR). The impact of social deprivation was reported in the 2003 UKRR Report [4].

There were substantial variations in the crude PCT/HB prevalence rate pmp, from $444 \mathrm{pmp}$ (Shetland, population 22,500) to $1,904 \mathrm{pmp}$ (Brent, population 256,300). There were similar variations in the standardised prevalence ratios (ratio of observed: expected prevalence rate given the age/gender breakdown of the PCT/HB) from 0.49 (Shetland) to 2.47 (Brent) (table 2.5). Confidence intervals are not presented for the rates per million population for 2011 but figures D3 and D4 in appendix D (www.renalreg.com) can be used to determine if a PCT/HB falls within the range representing the $95 \%$ confidence limit of the national average prevalence rate. The annual standardised prevalence ratios were inherently more stable than the annual standardised incidence ratios (chapter 1).

Factors associated with variation in standardised prevalence ratios (SPRs) in Primary Care Trusts (PCT) in England, Health and Social Care Areas $(H B)$ in Northern Ireland, Local Health Boards in Wales (HB) and Health Boards in Scotland (HB)

Geographical considerations and ethnicity were major factors contributing to the variation in SPRs (table 2.5). In 2011, there were $61 \mathrm{PCT} / \mathrm{HBs}$ with a significantly low SPR, 70 with a 'normal' SPR and 46 with a significantly high SPR. The areas with high and low SPRs have been fairly consistent over the last few years. They tend to reflect the demographics of the regions in question such that urban, ethnically diverse populations in areas of high social deprivation have the highest prevalence rates of renal replacement therapy. Mean SPRs were significantly higher in the $58 \mathrm{PCT} / \mathrm{HBs}$ with an ethnic minority population greater than $10 \%$ than in those with lower ethnic minority populations $(\mathrm{p}<0.001)$. The SPR (correlation coefficient $\mathrm{r}=0.89 \mathrm{p}<0.001$ ) was positively correlated with ethnicity. In 2011 for each $10 \%$ increase in ethnic minority population, the age standardised prevalence ratio increased by 0.24 . In figure 2.3, the relationship between the ethnic composition of a PCT/HB and its SPR is demonstrated.

Only six of the $118 \mathrm{PCT} / \mathrm{HBs}$ with ethnic minority populations of less than $10 \%$ had high SPRs: Abertawe 
Table 2.5. Prevalence of RRT and standardised prevalence ratios in PCT/HB areas

PCT/HB - PCT in England, Health and Social Care Areas in Northern Ireland, Local Health Boards in Wales and Health Boards in Scotland $\mathrm{O} / \mathrm{E}$ - standardised prevalence ratio. Ratio of observed:expected rate of RRT given the age and gender breakdown of the area

LCL - lower 95\% confidence limit

UCL - upper 95\% confidence limit

pmp - per million population

Blank cells - no data returned to the UKRR for that year

Areas with significantly low prevalence ratios in 2011 are italicised in greyed areas, those with significantly high prevalence ratios in 2011 are bold in greyed areas

$\%$ non-White-percentage of the PCT/HB population that is non-White, from 2001 census (revised by ONS to 2007 for England)

${ }^{*} \mathrm{O} / \mathrm{E}$ for combined years, 2006-2011

\begin{tabular}{|c|c|c|c|c|c|c|c|c|c|c|c|c|c|}
\hline UK area & Name & $\begin{array}{c}\text { Total } \\
\text { population }\end{array}$ & $\begin{array}{l}2006 \\
\mathrm{O} / \mathrm{E}\end{array}$ & $\begin{array}{l}2007 \\
\mathrm{O} / \mathrm{E}\end{array}$ & $\begin{array}{l}2008 \\
\mathrm{O} / \mathrm{E}\end{array}$ & $\begin{array}{c}2009 \\
\mathrm{O} / \mathrm{E}\end{array}$ & $\begin{array}{l}2010 \\
\mathrm{O} / \mathrm{E}\end{array}$ & $\begin{array}{l}2011 \\
\mathrm{O} / \mathrm{E}\end{array}$ & $\begin{array}{l}95 \% \\
\text { LCL }\end{array}$ & $\begin{array}{l}95 \% \\
\text { UCL }\end{array}$ & $\begin{array}{c}\text { Crude rate } \\
\text { pmp }\end{array}$ & $\mathbf{O} / \mathbf{E}^{*}$ & $\begin{array}{c}\% \\
\text { non- } \\
\text { White }\end{array}$ \\
\hline \multirow[t]{12}{*}{ North East } & County Durham & 510,800 & 0.91 & 0.90 & 0.86 & 0.85 & 0.85 & 0.85 & 0.77 & 0.94 & 764 & 0.87 & 2.5 \\
\hline & Darlington & 100,600 & 0.79 & 0.86 & 0.90 & 0.92 & 0.86 & 0.80 & 0.63 & 1.01 & 706 & 0.86 & 3.3 \\
\hline & Gateshead & 192,000 & 0.93 & 0.88 & 0.83 & 0.87 & 0.86 & 0.84 & 0.71 & 0.99 & 745 & 0.87 & 3.8 \\
\hline & Hartlepool & 91,400 & 0.97 & 0.88 & 0.92 & 0.91 & 0.84 & 0.82 & 0.65 & 1.05 & 711 & 0.89 & 2.6 \\
\hline & Middlesbrough & 142,100 & 1.06 & 1.04 & 1.06 & 1.07 & 1.07 & 1.08 & 0.91 & 1.29 & 866 & 1.06 & 8.6 \\
\hline & Newcastle & 292,200 & 0.93 & 0.96 & 0.97 & 0.93 & 0.87 & 0.85 & 0.74 & 0.98 & 647 & 0.92 & 9.7 \\
\hline & North Tyneside & 198,400 & 1.05 & 0.99 & 0.94 & 0.96 & 0.98 & 0.92 & 0.78 & 1.07 & 817 & 0.97 & 3.6 \\
\hline & Northumberland & 312,100 & 0.86 & 0.85 & 0.82 & 0.78 & 0.74 & 0.74 & 0.65 & 0.85 & 718 & 0.79 & 2.2 \\
\hline & Redcar and Cleveland & 137,300 & 1.02 & 1.01 & 1.00 & 0.99 & 0.94 & 0.99 & 0.83 & 1.18 & 903 & 0.99 & 3.0 \\
\hline & South Tyneside & 154,100 & 1.01 & 1.00 & 0.94 & 1.00 & 0.91 & 0.93 & 0.78 & 1.11 & 824 & 0.96 & 4.8 \\
\hline & Stockton-on-Tees Teaching & 192,600 & 0.87 & 0.81 & 0.81 & 0.79 & 0.78 & 0.83 & 0.70 & 0.98 & 706 & 0.81 & 4.7 \\
\hline & Sunderland Teaching & 283,400 & 0.94 & 0.94 & 0.97 & 0.95 & 0.94 & 0.91 & 0.80 & 1.03 & 787 & 0.94 & 3.3 \\
\hline \multirow[t]{24}{*}{ North West } & Ashton, Leigh and Wigan & 307,200 & 0.67 & 0.86 & 0.80 & 0.81 & 0.83 & 0.89 & 0.79 & 1.01 & 781 & 0.81 & 2.9 \\
\hline & Blackburn with Darwen Teaching & 140,000 & 1.19 & 1.38 & 1.29 & 1.30 & 1.27 & 1.31 & 1.11 & 1.55 & 993 & 1.29 & 22.7 \\
\hline & Blackpool & 140,200 & 0.59 & 0.76 & 0.79 & 0.84 & 0.80 & 0.82 & 0.68 & 0.99 & 742 & 0.77 & 3.7 \\
\hline & Bolton Teaching & 266,500 & 0.81 & 1.07 & 1.04 & 0.96 & 1.05 & 1.09 & 0.96 & 1.23 & 904 & 1.01 & 12.3 \\
\hline & Bury & 183,500 & 0.47 & 0.89 & 0.83 & 0.92 & 0.88 & 0.89 & 0.75 & 1.05 & 752 & 0.82 & 8.5 \\
\hline & Central and Eastern Cheshire & 457,200 & & 0.81 & 0.78 & 0.78 & 0.78 & 0.79 & 0.71 & 0.88 & 722 & 0.79 & 3.4 \\
\hline & Central Lancashire & 459,200 & 0.73 & 0.81 & 0.83 & 0.87 & 0.85 & 0.85 & 0.76 & 0.94 & 738 & 0.83 & 6.7 \\
\hline & Cumbria Teaching & 494,400 & 0.75 & 0.75 & 0.74 & 0.72 & 0.71 & 0.70 & 0.63 & 0.78 & 672 & 0.73 & 2.0 \\
\hline & East Lancashire Teaching & 381,200 & 0.94 & 1.08 & 1.03 & 1.00 & 0.97 & 0.97 & 0.87 & 1.09 & 837 & 1.00 & 9.4 \\
\hline & Halton and St Helens & 296,700 & 0.94 & 0.96 & 0.89 & 0.91 & 0.93 & 0.97 & 0.85 & 1.10 & 839 & 0.93 & 2.1 \\
\hline & Heywood, Middleton and Rochdale & 205,000 & & 1.01 & 1.02 & 1.06 & 0.99 & 1.03 & 0.89 & 1.20 & 844 & 1.02 & 12.6 \\
\hline & Knowsley & 149,200 & 1.16 & 1.11 & 1.05 & 1.02 & 0.94 & 0.92 & 0.77 & 1.11 & 764 & 1.03 & 2.8 \\
\hline & Liverpool & 445,300 & 1.16 & 1.12 & 1.13 & 1.13 & 1.10 & 1.11 & 1.00 & 1.22 & 874 & 1.12 & 8.3 \\
\hline & Manchester Teaching & 498,800 & & 1.04 & 1.11 & 1.14 & 1.17 & 1.16 & 1.05 & 1.28 & 766 & 1.13 & 23.4 \\
\hline & North Lancashire Teaching & 329,100 & 0.66 & 0.75 & 0.72 & 0.73 & 0.71 & 0.72 & 0.63 & 0.82 & 672 & 0.72 & 4.2 \\
\hline & Oldham & 219,600 & 0.61 & 0.94 & 0.95 & 0.94 & 0.92 & 0.91 & 0.78 & 1.06 & 733 & 0.88 & 12.2 \\
\hline & Salford & 229,100 & 0.64 & 0.79 & 0.85 & 0.83 & 0.85 & 0.85 & 0.73 & 0.99 & 672 & 0.81 & 7.7 \\
\hline & Sefton & 272,800 & 0.90 & 0.87 & 0.84 & 0.83 & 0.86 & 0.90 & 0.79 & 1.03 & 839 & 0.87 & 2.6 \\
\hline & Stockport & 284,700 & & 0.87 & 0.88 & 0.83 & 0.86 & 0.87 & 0.76 & 1.00 & 776 & 0.86 & 6.4 \\
\hline & Tameside and Glossop & 250,700 & & 1.03 & 0.99 & 0.98 & 0.99 & 0.99 & 0.87 & 1.14 & 842 & 1.00 & 5.9 \\
\hline & Trafford & 217,100 & & 0.78 & 0.74 & 0.76 & 0.87 & 0.82 & 0.70 & 0.96 & 700 & 0.80 & 11.2 \\
\hline & Warrington & 199,100 & 0.81 & 0.89 & 0.87 & 0.94 & 0.85 & 0.84 & 0.71 & 0.99 & 728 & 0.87 & 3.5 \\
\hline & Western Cheshire & 234,300 & 0.93 & 0.93 & 0.93 & 0.96 & 0.98 & 1.00 & 0.88 & 1.15 & 922 & 0.96 & 3.1 \\
\hline & Wirral & 308,800 & 1.04 & 0.96 & 0.89 & 0.85 & 0.82 & 0.82 & 0.72 & 0.93 & 735 & 0.89 & 2.8 \\
\hline \multirow{7}{*}{$\begin{array}{l}\text { Yorkshire } \\
\text { and the } \\
\text { Humber }\end{array}$} & Barnsley & 227,500 & 1.13 & 1.06 & 1.07 & 1.10 & 1.14 & 1.12 & 0.98 & 1.27 & 980 & 1.10 & 2.7 \\
\hline & Bradford and Airedale Teaching & 512,700 & 1.13 & 1.15 & 1.13 & 1.10 & 1.17 & 1.15 & 1.05 & 1.27 & 874 & 1.14 & 25.0 \\
\hline & Calderdale & 202,800 & 1.10 & 1.11 & 1.08 & 1.09 & 1.10 & 1.03 & 0.89 & 1.19 & 878 & 1.08 & 9.8 \\
\hline & Doncaster & 290,900 & 1.05 & 0.97 & 0.97 & 0.98 & 0.95 & 0.99 & 0.87 & 1.11 & 866 & 0.98 & 4.3 \\
\hline & East Riding of Yorkshire & 338,500 & 0.82 & 0.80 & 0.81 & 0.84 & 0.81 & 0.80 & 0.71 & 0.90 & 774 & 0.81 & 3.0 \\
\hline & Hull Teaching & 263,800 & 0.99 & 1.04 & 0.96 & 1.01 & 0.98 & 0.96 & 0.84 & 1.10 & 758 & 0.99 & 5.8 \\
\hline & Kirklees & 409,900 & 1.17 & 1.11 & 1.03 & 1.04 & 1.05 & 1.04 & 0.94 & 1.16 & 849 & 1.07 & 16.0 \\
\hline
\end{tabular}


Table 2.5. Continued

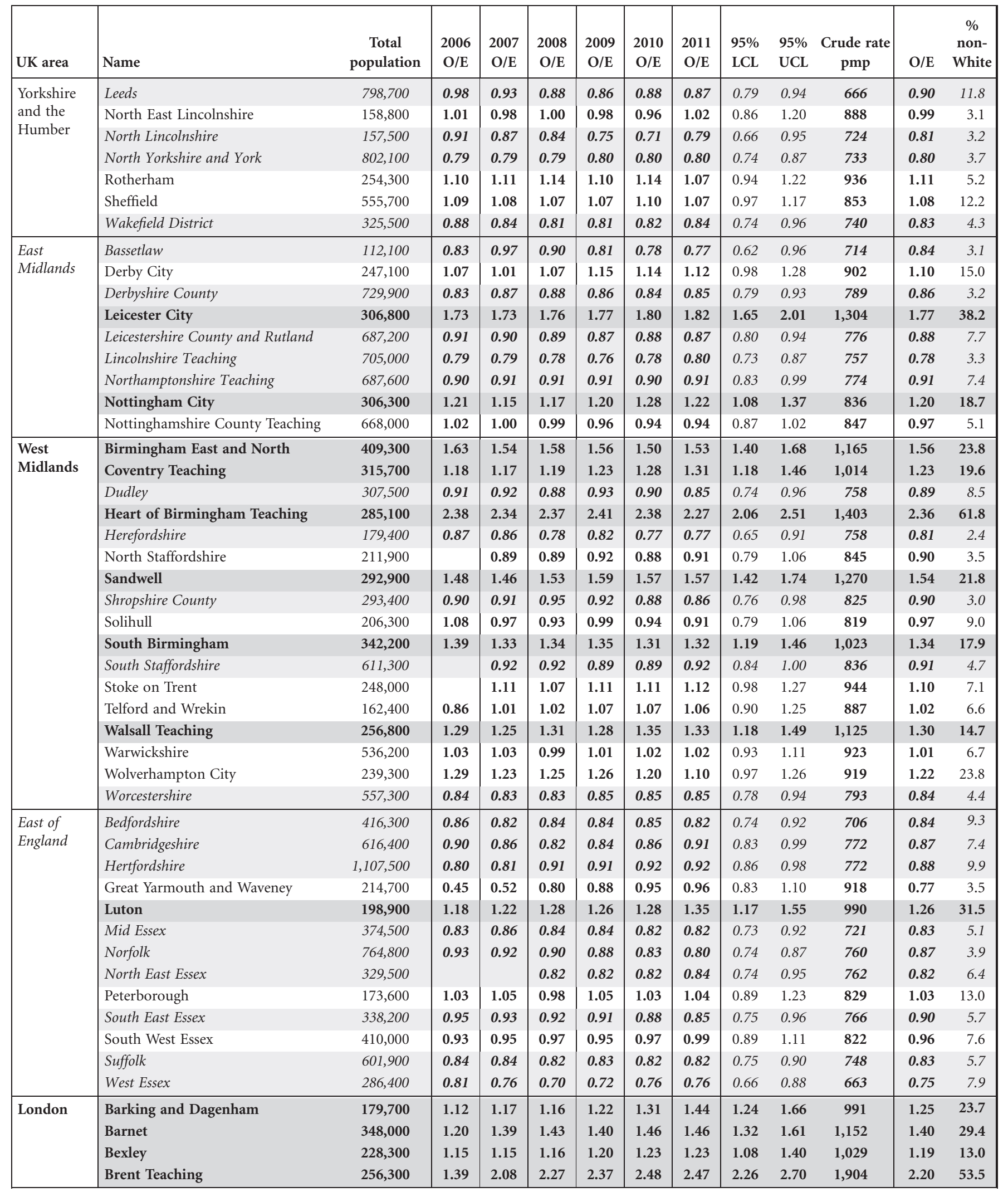


Table 2.5. Continued

\begin{tabular}{|c|c|c|c|c|c|c|c|c|c|c|c|c|c|}
\hline UK area & Name & $\begin{array}{c}\text { Total } \\
\text { population }\end{array}$ & $\begin{array}{l}2006 \\
\mathrm{O} / \mathrm{E}\end{array}$ & $\begin{array}{l}2007 \\
\mathrm{O} / \mathrm{E}\end{array}$ & $\begin{array}{c}2008 \\
\mathrm{O} / \mathrm{E}\end{array}$ & $\begin{array}{l}2009 \\
\mathrm{O} / \mathrm{E}\end{array}$ & $\begin{array}{l}2010 \\
\mathrm{O} / \mathrm{E}\end{array}$ & $\begin{array}{l}2011 \\
\mathrm{O} / \mathrm{E}\end{array}$ & $\begin{array}{l}95 \% \\
\text { LCL }\end{array}$ & $\begin{array}{l}95 \% \\
\text { UCL }\end{array}$ & $\begin{array}{c}\text { Crude rate } \\
\text { pmp }\end{array}$ & $\mathrm{O} / \mathrm{E}$ & $\begin{array}{c}\% \\
\text { non- } \\
\text { White }\end{array}$ \\
\hline \multirow[t]{27}{*}{ London } & Bromley & 312,400 & 1.01 & 0.97 & 1.02 & 0.98 & 1.02 & 1.01 & 0.89 & 1.14 & 861 & 1.00 & 11.9 \\
\hline & Camden & 235,500 & 1.04 & 1.11 & 1.16 & 1.21 & 1.24 & 1.26 & 1.10 & 1.45 & 883 & 1.17 & 24.9 \\
\hline & City and Hackney Teaching & 231,000 & 1.36 & 1.40 & 1.33 & 1.40 & 1.50 & 1.57 & 1.38 & 1.78 & 1,056 & 1.43 & 35.7 \\
\hline & Croydon & 345,400 & 1.14 & 1.31 & 1.31 & 1.37 & 1.36 & 1.42 & 1.28 & 1.57 & 1,123 & 1.32 & 34.5 \\
\hline & Ealing & 318,300 & 1.45 & 1.60 & 1.90 & 1.91 & 1.95 & 1.92 & 1.75 & 2.10 & 1,448 & 1.80 & 40.7 \\
\hline & Enfield & 295,000 & 1.46 & 1.41 & 1.42 & 1.40 & 1.42 & 1.53 & 1.38 & 1.70 & 1,193 & 1.44 & 28.0 \\
\hline & Greenwich Teaching & 228,100 & 1.10 & 1.14 & 1.23 & 1.26 & 1.40 & 1.43 & 1.26 & 1.63 & 1,035 & 1.27 & 26.1 \\
\hline & Hammersmith and Fulham & 169,800 & 1.23 & 1.24 & 1.27 & 1.35 & 1.34 & 1.38 & 1.19 & 1.61 & 995 & 1.30 & 21.0 \\
\hline & Haringey Teaching & 225,100 & 1.50 & 1.52 & 1.58 & 1.57 & 1.59 & 1.73 & 1.54 & 1.95 & 1,253 & 1.59 & 33.1 \\
\hline & Harrow & 230,300 & & 1.49 & 1.68 & 1.76 & 1.83 & 1.89 & 1.70 & 2.10 & 1,524 & 1.74 & 44.7 \\
\hline & Havering & 236,100 & & 0.80 & 0.81 & 0.82 & 0.80 & 0.85 & 0.73 & 0.98 & 737 & 0.82 & 8.8 \\
\hline & Hillingdon & 266,200 & 1.08 & 0.94 & 1.31 & 1.32 & 1.33 & 1.41 & 1.25 & 1.58 & 1,082 & 1.24 & 25.9 \\
\hline & Hounslow & 236,700 & 1.25 & 1.27 & 1.47 & 1.51 & 1.57 & 1.64 & 1.46 & 1.84 & 1,221 & 1.46 & 37.8 \\
\hline & Islington & 193,900 & 1.45 & 1.36 & 1.29 & 1.31 & 1.41 & 1.47 & 1.28 & 1.69 & 1,016 & 1.38 & 22.9 \\
\hline & Kensington and Chelsea & 169,500 & & 0.80 & 0.98 & 0.97 & 1.00 & 0.99 & 0.84 & 1.17 & 814 & 0.95 & 22.6 \\
\hline & Kingston & 169,000 & & 1.04 & 1.15 & 1.12 & 1.10 & 1.11 & 0.94 & 1.30 & 846 & 1.11 & 19.9 \\
\hline & Lambeth & 284,400 & 1.32 & 1.60 & 1.59 & 1.66 & 1.63 & 1.71 & 1.53 & 1.90 & 1,181 & 1.59 & 32.0 \\
\hline & Lewisham & 266,400 & 1.62 & 1.66 & 1.63 & 1.71 & 1.66 & 1.72 & 1.55 & 1.92 & 1,239 & 1.67 & 34.4 \\
\hline & Newham & 240,200 & 1.77 & 1.82 & 1.84 & 1.90 & 2.13 & 2.26 & 2.03 & 2.51 & 1,457 & 1.97 & 57.0 \\
\hline & Redbridge & 270,300 & 1.18 & 1.18 & 1.31 & 1.37 & 1.45 & 1.42 & 1.27 & 1.60 & 1,080 & 1.33 & 40.9 \\
\hline & Richmond and Twickenham & 190,800 & & 0.62 & 0.69 & 0.74 & 0.76 & 0.76 & 0.64 & 0.91 & 618 & 0.72 & 11.7 \\
\hline & Southwark & 287,100 & 1.46 & 1.58 & 1.61 & 1.63 & 1.68 & 1.78 & 1.61 & 1.98 & 1,247 & 1.63 & 34.1 \\
\hline & Sutton and Merton & 403,000 & & 1.13 & 1.16 & 1.21 & 1.23 & 1.23 & 1.12 & 1.36 & 965 & 1.20 & 20.8 \\
\hline & Tower Hamlets & 238,100 & 1.13 & 1.22 & 1.27 & 1.40 & 1.46 & 1.51 & 1.33 & 1.73 & 932 & 1.34 & 22.8 \\
\hline & Waltham Forest & 227,400 & 1.42 & 1.59 & 1.56 & 1.53 & 1.62 & 1.73 & 1.54 & 1.95 & 1,240 & 1.58 & 36.6 \\
\hline & Wandsworth & 289,200 & & 1.36 & 1.37 & 1.45 & 1.43 & 1.40 & 1.25 & 1.58 & 968 & 1.40 & 19.7 \\
\hline & Westminster & 253,400 & & 0.89 & 0.99 & 1.08 & 1.10 & 1.18 & 1.04 & 1.35 & 888 & 1.05 & 27.8 \\
\hline \multirow{8}{*}{$\begin{array}{l}\text { South East } \\
\text { Coast }\end{array}$} & Brighton and Hove City & 258,400 & 0.87 & 0.87 & 0.87 & 0.86 & 0.85 & 0.86 & 0.74 & 0.99 & 670 & 0.86 & 8.7 \\
\hline & East Sussex Downs and Weald & 336,100 & 0.77 & 0.79 & 0.74 & 0.70 & 0.70 & 0.68 & 0.60 & 0.78 & 661 & 0.73 & 4.9 \\
\hline & Eastern and Coastal Kent & 742,200 & & 0.86 & 0.93 & 0.94 & 0.96 & 0.95 & 0.88 & 1.03 & 847 & 0.93 & 5.3 \\
\hline & Hastings and Rother & 179,700 & 0.80 & 0.76 & 0.78 & 0.73 & 0.78 & 0.75 & 0.63 & 0.89 & 729 & 0.77 & 5.2 \\
\hline & Medway & 256,600 & & 0.85 & 0.90 & 0.91 & 0.88 & 0.91 & 0.79 & 1.04 & 740 & 0.89 & 7.5 \\
\hline & Surrey & $1,114,400$ & 0.77 & 0.85 & 0.87 & 0.88 & 0.89 & 0.88 & 0.82 & 0.94 & 767 & 0.86 & 8.3 \\
\hline & West Kent & 685,100 & & 0.85 & 0.88 & 0.89 & 0.86 & 0.85 & 0.78 & 0.93 & 744 & 0.87 & 6.8 \\
\hline & West Sussex & 800,000 & 0.75 & 0.81 & 0.82 & 0.82 & 0.82 & 0.80 & 0.74 & 0.86 & 740 & 0.80 & 5.8 \\
\hline \multirow{9}{*}{$\begin{array}{l}\text { South } \\
\text { Central }\end{array}$} & Berkshire East & 406,500 & 1.01 & 1.14 & 1.13 & 1.16 & 1.21 & 1.22 & 1.11 & 1.35 & 962 & 1.15 & 18.9 \\
\hline & Berkshire West & 471,500 & 1.01 & 1.09 & 1.09 & 1.10 & 1.04 & 1.03 & 0.93 & 1.14 & 829 & 1.06 & 10.1 \\
\hline & Buckinghamshire & 512,100 & 0.97 & 0.95 & 0.94 & 0.93 & 0.91 & 0.87 & 0.79 & 0.96 & 760 & 0.93 & 10.4 \\
\hline & Hampshire & $1,297,200$ & 0.79 & 0.77 & 0.79 & 0.81 & 0.80 & 0.79 & 0.74 & 0.84 & 713 & 0.79 & 4.2 \\
\hline & Isle of Wight National Health Service & 140,200 & 0.62 & 0.58 & 0.57 & 0.54 & 0.55 & 0.59 & 0.47 & 0.73 & 585 & 0.57 & 3.6 \\
\hline & Milton Keynes & 247,000 & 0.84 & 0.91 & 0.92 & 0.90 & 0.92 & 0.94 & 0.81 & 1.08 & 737 & 0.91 & 12.7 \\
\hline & Oxfordshire & 624,200 & 1.03 & 0.95 & 0.91 & 0.89 & 0.89 & 0.92 & 0.84 & 1.01 & 764 & 0.93 & 8.1 \\
\hline & Portsmouth City Teaching & 207,200 & 0.98 & 0.97 & 0.96 & 0.92 & 0.90 & 0.95 & 0.81 & 1.12 & 709 & 0.94 & 8.0 \\
\hline & Southampton City & 239,800 & 0.89 & 0.90 & 0.94 & 0.93 & 0.99 & 1.01 & 0.87 & 1.17 & 742 & 0.95 & 11.4 \\
\hline \multirow[t]{6}{*}{ South West } & Bath and North East Somerset & 179,800 & 0.91 & 0.91 & 0.84 & 0.86 & 0.85 & 0.79 & 0.67 & 0.95 & 673 & 0.86 & 5.8 \\
\hline & Bournemouth and Poole Teaching & 310,800 & 0.86 & 0.88 & 0.87 & 0.84 & 0.82 & 0.81 & 0.71 & 0.93 & 718 & 0.84 & 5.0 \\
\hline & Bristol & 441,100 & 1.28 & 1.21 & 1.25 & 1.22 & 1.19 & 1.20 & 1.08 & 1.32 & 886 & 1.22 & 11.6 \\
\hline & Cornwall and Isles of Scilly & 537,900 & 1.05 & 1.00 & 0.98 & 0.98 & 0.95 & 0.92 & 0.84 & 1.00 & 881 & 0.98 & 2.8 \\
\hline & Devon & 749,700 & 0.82 & 0.83 & 0.85 & 0.87 & 0.86 & 0.85 & 0.79 & 0.92 & 823 & 0.85 & 3.3 \\
\hline & Dorset & 404,900 & 0.81 & 0.83 & 0.85 & 0.85 & 0.83 & 0.79 & 0.70 & 0.88 & 800 & 0.83 & 3.5 \\
\hline
\end{tabular}


Table 2.5. Continued

\begin{tabular}{|c|c|c|c|c|c|c|c|c|c|c|c|c|c|}
\hline UK area & Name & $\begin{array}{c}\text { Total } \\
\text { population }\end{array}$ & $\begin{array}{c}2006 \\
\mathrm{O} / \mathrm{E}\end{array}$ & $\begin{array}{l}2007 \\
\mathrm{O} / \mathrm{E}\end{array}$ & $\begin{array}{c}2008 \\
\mathrm{O} / \mathrm{E}\end{array}$ & $\begin{array}{l}2009 \\
\mathrm{O} / \mathrm{E}\end{array}$ & $\begin{array}{c}2010 \\
\mathrm{O} / \mathrm{E}\end{array}$ & $\begin{array}{c}2011 \\
\mathrm{O} / \mathrm{E}\end{array}$ & $\begin{array}{l}95 \% \\
\text { LCL }\end{array}$ & $\begin{array}{l}95 \% \\
\text { UCL }\end{array}$ & $\begin{array}{c}\text { Crude rate } \\
\text { pmp }\end{array}$ & $\mathrm{O} / \mathrm{E}$ & $\begin{array}{c}\% \\
\text { non- } \\
\text { White }\end{array}$ \\
\hline \multirow[t]{8}{*}{ South West } & Gloucestershire & 593,600 & 0.91 & 0.87 & 0.82 & 0.85 & 0.85 & 0.85 & 0.78 & 0.94 & 773 & 0.86 & 4.7 \\
\hline & North Somerset & 212,100 & 0.97 & 0.91 & 0.91 & 0.86 & 0.84 & 0.84 & 0.72 & 0.98 & 787 & 0.89 & 3.6 \\
\hline & Plymouth Teaching & 258,900 & 1.18 & 1.15 & 1.12 & 1.12 & 1.15 & 1.15 & 1.01 & 1.30 & 927 & 1.14 & 4.4 \\
\hline & Somerset & 525,500 & 0.87 & 0.83 & 0.81 & 0.81 & 0.84 & 0.85 & 0.78 & 0.94 & 811 & 0.83 & 3.2 \\
\hline & South Gloucestershire & 264,900 & 1.04 & 0.99 & 0.98 & 0.92 & 0.97 & 0.94 & 0.82 & 1.08 & 812 & 0.97 & 5.0 \\
\hline & Swindon & 206,900 & 0.93 & 0.88 & 0.86 & 0.88 & 0.92 & 0.95 & 0.81 & 1.10 & 778 & 0.90 & 7.1 \\
\hline & Torbay & 134,400 & 0.86 & 0.81 & 0.90 & 0.85 & 0.91 & 0.94 & 0.79 & 1.12 & 915 & 0.88 & 3.1 \\
\hline & Wiltshire & 459,800 & 0.69 & 0.72 & 0.74 & 0.73 & 0.73 & 0.74 & 0.66 & 0.83 & 668 & 0.73 & 3.4 \\
\hline \multirow[t]{7}{*}{ Wales } & Betsi Cadwaladr University & 678,500 & 1.00 & 0.95 & 0.94 & 0.91 & 0.88 & 0.85 & 0.78 & 0.93 & 789 & 0.92 & 1.0 \\
\hline & Powys Teaching & 131,100 & 0.92 & 0.87 & 0.88 & 0.93 & 0.88 & 0.86 & 0.71 & 1.03 & 862 & 0.89 & 0.9 \\
\hline & Hywel Dda & 374,800 & 1.02 & 0.95 & 1.00 & 0.95 & 0.90 & 0.92 & 0.83 & 1.03 & 872 & 0.95 & 1.0 \\
\hline & Abertawe Bro Morgannwg University & 504,800 & 1.26 & 1.26 & 1.19 & 1.22 & 1.26 & 1.24 & 1.14 & 1.35 & 1,099 & 1.24 & 1.6 \\
\hline & Cwm Taf & 290,600 & 1.46 & 1.52 & 1.43 & 1.40 & 1.31 & 1.35 & 1.22 & 1.51 & 1,163 & 1.41 & 1.1 \\
\hline & Aneurin Bevan & 561,300 & 1.16 & 1.17 & 1.11 & 1.09 & 1.12 & 1.10 & 1.01 & 1.20 & 967 & 1.12 & 1.9 \\
\hline & Cardiff and Vale University & 466,100 & 1.18 & 1.18 & 1.08 & 1.08 & 1.07 & 1.06 & 0.96 & 1.17 & 824 & 1.10 & 6.7 \\
\hline \multirow[t]{14}{*}{ Scotland } & Ayrshire \& Arran & 366,900 & 1.22 & 1.14 & 1.14 & 1.08 & 1.07 & 1.02 & 0.92 & 1.13 & 940 & 1.11 & 0.7 \\
\hline & Borders & 113,000 & 0.86 & 0.96 & 0.99 & 1.02 & 1.06 & 0.95 & 0.79 & 1.15 & 920 & 0.98 & 0.6 \\
\hline & Dumfries and Galloway & 148,100 & 1.03 & 0.95 & 0.96 & 0.93 & 0.89 & 0.86 & 0.73 & 1.03 & 858 & 0.93 & 0.7 \\
\hline & Fife & 364,800 & 1.00 & 0.97 & 0.96 & 0.94 & 0.95 & 0.99 & 0.89 & 1.10 & 874 & 0.97 & 1.3 \\
\hline & Forth Valley & 293,100 & 0.93 & 0.98 & 0.95 & 0.92 & 0.94 & 0.89 & 0.78 & 1.01 & 771 & 0.93 & 1.1 \\
\hline & Grampian & 550,500 & 1.05 & 1.01 & 0.99 & 0.96 & 0.95 & 0.95 & 0.86 & 1.04 & 830 & 0.98 & 1.6 \\
\hline & Greater Glasgow \& Clyde & $1,204,100$ & 1.21 & 1.17 & 1.13 & 1.09 & 1.06 & 1.05 & 0.99 & 1.12 & 879 & 1.11 & 3.4 \\
\hline & Highland & 310,700 & 1.13 & 1.12 & 1.06 & 1.04 & 1.00 & 0.91 & 0.81 & 1.03 & 872 & 1.04 & 0.8 \\
\hline & Lanarkshire & 562,700 & 1.07 & 0.99 & 0.97 & 0.96 & 0.96 & 0.93 & 0.85 & 1.02 & 803 & 0.98 & 1.2 \\
\hline & Lothian & 837,000 & 0.98 & 0.95 & 0.92 & 0.89 & 0.85 & 0.81 & 0.74 & 0.88 & 668 & 0.90 & 2.8 \\
\hline & Orkney & 19,800 & 1.15 & 0.95 & 1.14 & 1.09 & 0.99 & 0.84 & 0.52 & 1.38 & 808 & 1.02 & 0.4 \\
\hline & Shetland & 22,500 & 0.50 & 0.71 & 0.50 & 0.54 & 0.57 & 0.49 & 0.27 & 0.92 & 444 & 0.55 & 1.1 \\
\hline & Tayside & 402,400 & 1.22 & 1.14 & 1.06 & 1.07 & 1.04 & 1.02 & 0.92 & 1.13 & 924 & 1.09 & 1.9 \\
\hline & Western Isles & 26,500 & 0.54 & 0.83 & 0.75 & 0.71 & 0.80 & 0.65 & 0.41 & 1.05 & 642 & 0.72 & 0.6 \\
\hline \multirow{5}{*}{$\begin{array}{l}\text { Northern } \\
\text { Ireland }\end{array}$} & Belfast & 335,700 & 1.38 & 1.37 & 1.31 & 1.22 & 1.21 & 1.17 & 1.05 & 1.31 & 923 & 1.27 & 1.1 \\
\hline & Northern & 458,600 & 1.20 & 1.15 & 1.11 & 1.05 & 1.01 & 1.05 & 0.95 & 1.16 & 859 & 1.09 & 0.6 \\
\hline & Southern & 357,700 & 1.05 & 0.99 & 1.00 & 0.98 & 1.00 & 1.04 & 0.92 & 1.17 & 788 & 1.01 & 0.4 \\
\hline & South Eastern & 347,100 & 1.06 & 1.01 & 1.00 & 0.96 & 0.89 & 0.91 & 0.81 & 1.03 & 755 & 0.97 & 0.7 \\
\hline & Western & 299,900 & 1.16 & 1.14 & 1.10 & 1.13 & 1.12 & 1.08 & 0.95 & 1.22 & 830 & 1.12 & 0.5 \\
\hline
\end{tabular}

Bro Morgannwg University, Aneurin Bevan, Belfast, Cwm Taf, Plymouth and Liverpool. Forty (69.0\%) of the $58 \mathrm{PCT} / \mathrm{HBs}$ with ethnic minority populations greater than $10 \%$ had high SPRs, whereas only 4 (6.9\%) (Trafford, Leeds, Richmond \& Twickenham, Buckinghamshire) had low SPRs. However, not all PCT/HBs with a high $(>15 \%)$ ethnic minority population also had higher than expected RRT prevalence rates. For example Kingston and Kensington had rates similar to average (1.11 and 0.95 respectively 20062011), possibly explained by lower levels of social deprivation in these areas. The standardised prevalence ratios in each region of England and in Wales, Northern Ireland and Scotland are presented in table 2.6. North
East England, North West England, Yorkshire and Humber, East Midlands, East of England, South East England, South Central and South West England had lower than expected prevalence rates of RRT given the age and gender of their populations and this pattern has been similar for the last five years. West Midlands, London and Wales had higher than expected prevalence rates of RRT given the age and gender of their populations and again this pattern has remained similar for the last five years. Scotland and Northern Ireland previously had higher than expected prevalence rates but in more recent years were similar to their expected rates. There was marked variation (17-fold) in prevalence rates in over 80 year olds between PCT/HBs (data not shown). 


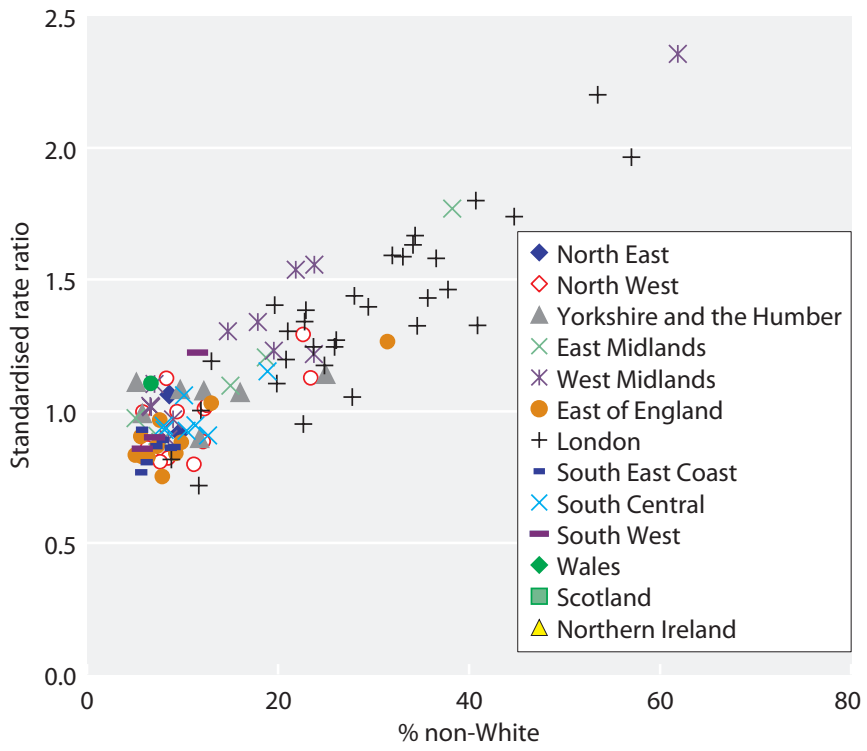

Fig. 2.3. Ethnicity and standardised prevalence ratios for all PCT/ $\mathrm{HB}$ areas by percentage non-White on 31/12/2011 (excluding areas with $<5 \%$ ethnic minorities)

$\mathrm{SPR}=$ standardised prevalence ratio

\section{Case mix in prevalent RRT patients}

Time on RRT (vintage)

Table 2.7 shows the median time, in years, since starting RRT of prevalent RRT patients on 31/12/2011. Median time on RRT for all prevalent patients remained static at 5.6 years (for patients who recovered for $>90$ days and then subsequently restarted RRT the median time from the start of RRT was calculated from the most recent start date). Patients with functioning transplants had survived a median of 10.3 years on RRT whilst the
Table 2.7. Median time on RRT of prevalent patients on $31 / 12 / 2011$

\begin{tabular}{lrc}
\hline Modality & $\mathrm{N}$ & $\begin{array}{c}\text { Median time treated } \\
\text { (years) }\end{array}$ \\
\hline Haemodialysis & 22,706 & 3.3 \\
Peritoneal dialysis & 3,768 & 1.8 \\
Transplant & 25,014 & 10.3 \\
All RRT & $\mathbf{5 1 , 4 8 8}$ & $\mathbf{5 . 6}$
\end{tabular}

All patients without a treatment modality were excluded

Median time on RRT was calculated from the most recent start date Patients with an initial treatment modality of transferred in or transferred out were excluded from the calculation of median time on RRT, since their treatment start date was not accurately known

median time on RRT of HD and PD patients was significantly less (3.3 and 1.8 years respectively, $\mathrm{p}<0.001$ ).

\section{Age}

The median age of prevalent UK patients on RRT at 31st December 2011 was slightly higher (58.2 years) compared with 2010 (57.9 years) (table 2.8) and significantly higher than in 2005 when it was 55 years. There were marked differences between modalities; the median age of HD patients (66.5 years) was greater than that of those on PD (62.7 years) and substantially higher than that of transplanted patients (51.7 years). About half (50.1\%) of the UK prevalent RRT population was in the 40-64 years age group. Northern Ireland and Wales had a higher proportion of patients aged 75 years and older (16.9\% and $17.2 \%$ respectively) compared with England (15.6\%) and Scotland (13.6\%) (table 2.9). Furthermore, there existed a wide range between centres

Table 2.6. Standardised rate ratio of RRT for each Strategic Health Authority in England and for Wales, Scotland and Northern Ireland in 2011

\begin{tabular}{|c|c|c|c|c|c|}
\hline UK Area & Total population & $\mathrm{O} / \mathrm{E}$ & $95 \% \mathrm{LCL}$ & 95\% UCL & Crude rate pmp \\
\hline North West & $6,969,700$ & 0.91 & 0.89 & 0.93 & 778.5 \\
\hline East Midlands & $4,450,000$ & 0.95 & 0.92 & 0.99 & 831.5 \\
\hline West Midlands & $5,455,000$ & 1.12 & 1.09 & 1.15 & 955.6 \\
\hline East of England & $5,832,700$ & 0.88 & 0.86 & 0.91 & 771.7 \\
\hline South Central & $4,145,700$ & 0.90 & 0.87 & 0.94 & 762.7 \\
\hline South West & $5,280,300$ & 0.89 & 0.86 & 0.91 & 804.5 \\
\hline Wales & $3,007,200$ & 1.05 & 1.01 & 1.09 & 929.4 \\
\hline Scotland & $5,222,100$ & 0.95 & 0.92 & 0.98 & 829.7 \\
\hline Northern Ireland & $1,799,000$ & 1.05 & 1.00 & 1.10 & 832.1 \\
\hline
\end{tabular}

$\mathrm{O} / \mathrm{E}$ - observed/expected prevalence rate ratio given the age/gender breakdown of each region

Bold - higher than expected prevalence rate ratio 
Table 2.8. Median age of prevalent RRT patients by treatment modality in renal centres on 31/12/2011

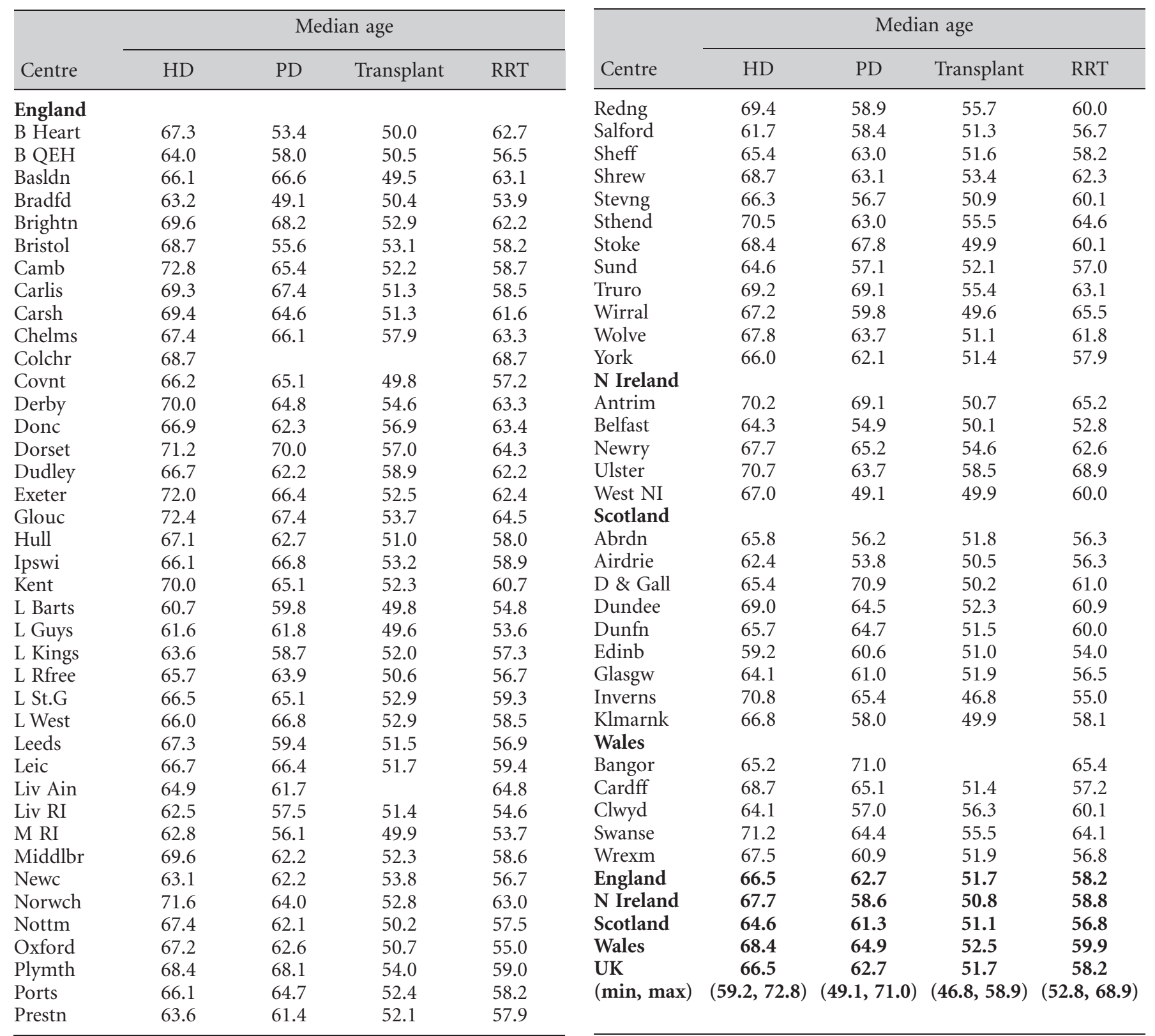

Blank cells indicate no patients on that treatment modality attending that centre when data were collected

in the proportion of patients aged over 75 ( $8.4 \%$ in Edinburgh to $32.8 \%$ in Ulster).

There was inter-centre variation in the median age of patients on RRT. Ulster had the highest median age (68.9 years), whilst Belfast had the lowest median age (52.8 years) (table 2.8). This likely reflects either ethnic make up of the catchment populations or follow up of younger transplant patients (as above in the case of Belfast). The median age of the non-White dialysis population was lower than the overall dialysis population
(60.6 vs. 65.9 years, data not shown). The differing age distributions of the transplant and dialysis populations are illustrated in figure 2.4, demonstrating that the age peak for prevalent dialysis patients is 27 years later than for prevalent transplant patients.

In the UK on 31st December 2011, 62.7\% of patients aged less than 65 years on RRT had a functioning transplant (table 2.15), compared with only $25.0 \%$ aged 65 years and over. There was a similar pattern in all four UK countries. 
Table 2.9. Percentage of prevalent RRT patients in each age group by centre on $31 / 12 / 2011$

\begin{tabular}{|c|c|c|c|c|c|}
\hline \multirow[b]{2}{*}{ Centre } & \multirow[b]{2}{*}{$\mathrm{N}$} & \multicolumn{4}{|c|}{ Percentage of patients } \\
\hline & & $18-39$ years & 40-64 years & 65-74 years & $75+$ years \\
\hline B Heart & 666 & 12.3 & 41.9 & 25.2 & 20.6 \\
\hline B QEH & 1,923 & 16.3 & 51.7 & 16.7 & 15.3 \\
\hline Basldn & 238 & 13.4 & 40.8 & 21.4 & 24.4 \\
\hline Bristol & 1,311 & 16.1 & 48.5 & 19.8 & 15.6 \\
\hline Camb & 1,086 & 15.1 & 50.7 & 17.8 & 16.4 \\
\hline Carlis & 219 & 13.7 & 51.1 & 22.8 & 12.3 \\
\hline Carsh & 1,410 & 11.4 & 46.2 & 22.1 & 20.3 \\
\hline Chelms & 216 & 8.8 & 46.8 & 20.8 & 23.6 \\
\hline Colchr & 120 & 5.8 & 30.8 & 31.7 & 31.7 \\
\hline Dudley & 287 & 8.4 & 48.1 & 24.4 & 19.2 \\
\hline Exeter & 813 & 10.6 & 45.3 & 20.5 & 23.6 \\
\hline Glouc & 390 & 9.2 & 42.1 & 24.4 & 24.4 \\
\hline Hull & 764 & 13.9 & 51.2 & 19.6 & 15.3 \\
\hline Ipswi & 340 & 13.2 & 53.5 & 20.3 & 12.9 \\
\hline Kent $^{*}$ & 849 & 12.5 & 45.1 & 24.7 & 17.7 \\
\hline L Barts & 1,900 & 17.7 & 54.5 & 16.4 & 11.3 \\
\hline L Guys & 1,680 & 20.1 & 53.8 & 15.2 & 10.8 \\
\hline L Kings & 882 & 13.4 & 51.9 & 20.0 & 14.7 \\
\hline L Rfree & 1,773 & 18.2 & 49.6 & 17.7 & 14.5 \\
\hline L St.G & 719 & 14.0 & 50.8 & 19.6 & 15.6 \\
\hline Newc & 916 & 16.0 & 53.6 & 21.0 & 9.4 \\
\hline Norwch & 612 & 12.9 & 40.4 & 23.0 & 23.7 \\
\hline Nottm & 1,019 & 17.1 & 49.2 & 19.2 & 14.5 \\
\hline Oxford & 1,444 & 17.5 & 52.0 & 17.2 & 13.4 \\
\hline Plymth & 465 & 13.1 & 48.8 & 23.2 & 14.8 \\
\hline Ports & 1,394 & 14.3 & 51.5 & 20.3 & 13.8 \\
\hline Prestn & 1,023 & 13.5 & 52.1 & 21.0 & 13.4 \\
\hline Redng & 688 & 11.6 & 49.9 & 20.3 & 18.2 \\
\hline Salford & 846 & 14.8 & 54.1 & 18.7 & 12.4 \\
\hline Sheff & 1,260 & 14.0 & 51.3 & 19.0 & 15.7 \\
\hline Shrew & 342 & 11.4 & 44.2 & 21.1 & 23.4 \\
\hline Stevng & 638 & 12.1 & 46.9 & 20.8 & 20.2 \\
\hline Sthend & 214 & 13.6 & 39.3 & 22.4 & 24.8 \\
\hline Stoke & 695 & 15.1 & 45.2 & 20.0 & 19.7 \\
\hline Sund & 390 & 14.9 & 53.6 & 19.0 & 12.6 \\
\hline Truro & 357 & 12.0 & 44.3 & 21.3 & 22.4 \\
\hline Wirral & 241 & 9.1 & 39.8 & 23.7 & 27.4 \\
\hline Wolve & 516 & 10.7 & 46.3 & 23.1 & 20.0 \\
\hline York $^{*}$ & 339 & 18.9 & 44.5 & 19.8 & 16.8 \\
\hline
\end{tabular}


Table 2.9. Continued

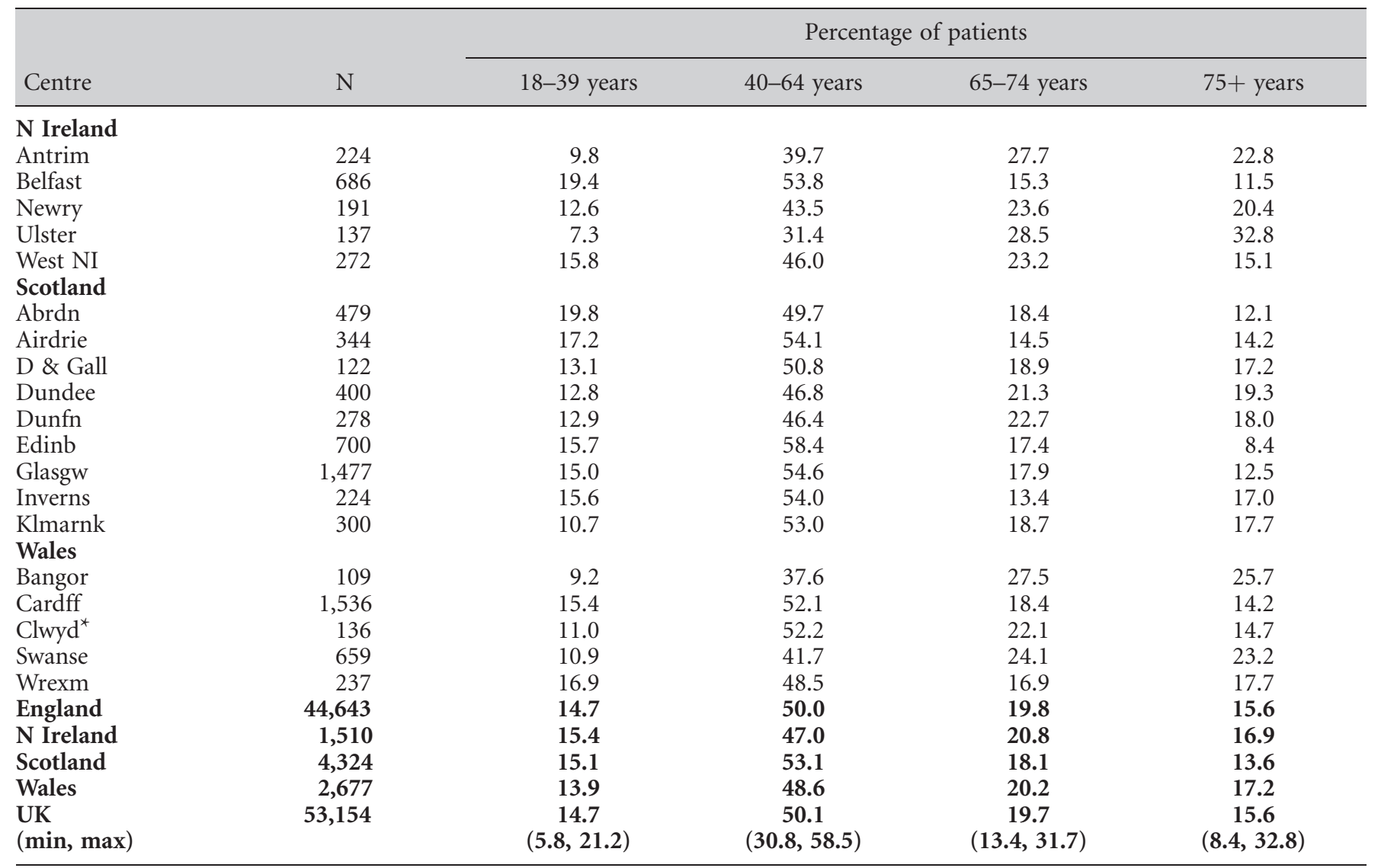

* 16 transplant patients from Kent, 21 transplant patients from Leeds, 27 transplant patients from York, were not included in this analysis. 6 transplant patients, $13 \mathrm{HD}$ patients and $12 \mathrm{PD}$ patients from Clwyd were not included in this analysis

Gender

Standardising the age of the UK RRT prevalent patients, by using the age and gender distribution of the UK population by PCT/HB (from ONS mid-2010

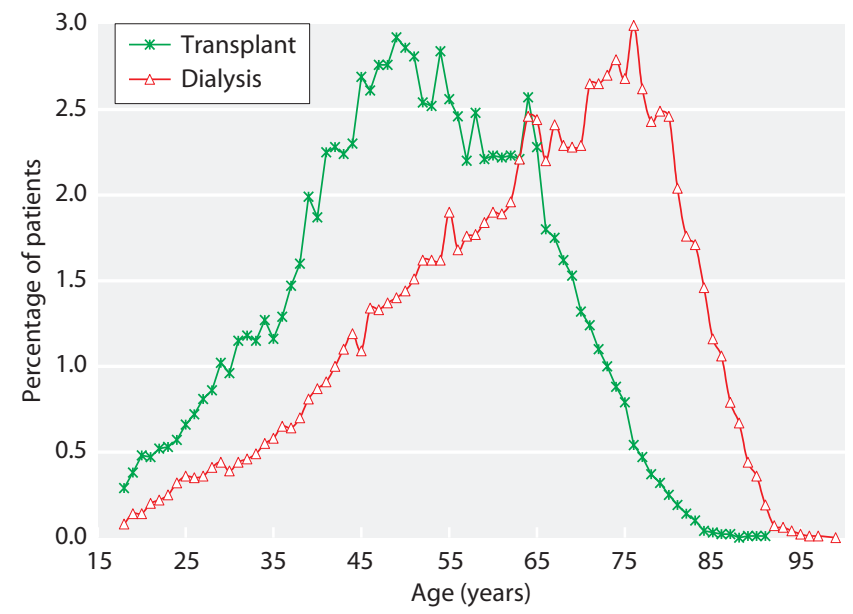

Fig. 2.4. Age profile of prevalent RRT patients by modality on $31 / 12 / 2011$ population estimates), allowed estimation of crude prevalence rates by age and gender (figure 2.5). This shows a progressive increase in prevalence rate with age, peaking at 2,099 pmp (a slight increase from

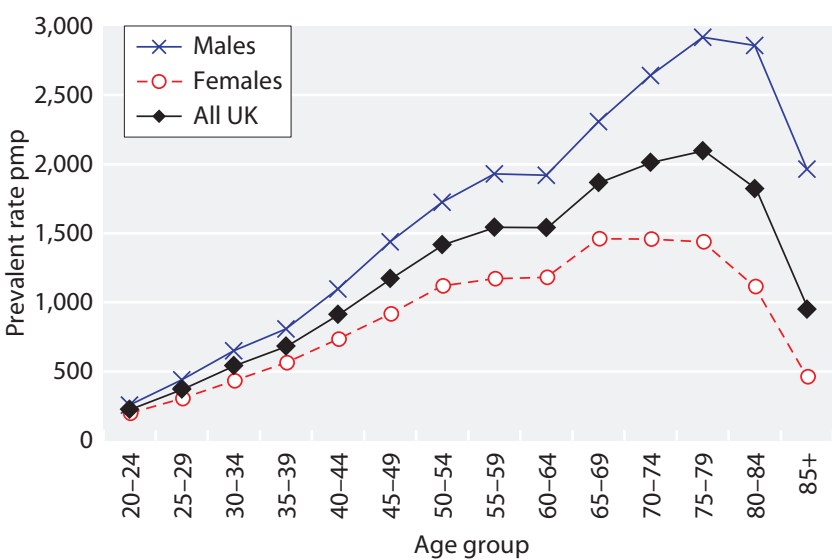

Fig. 2.5. Prevalence rate of RRT patients per million population by age and gender on 31/12/2011 
Table 2.10. Ethnicity of prevalent RRT patients by centre on 31/12/2011

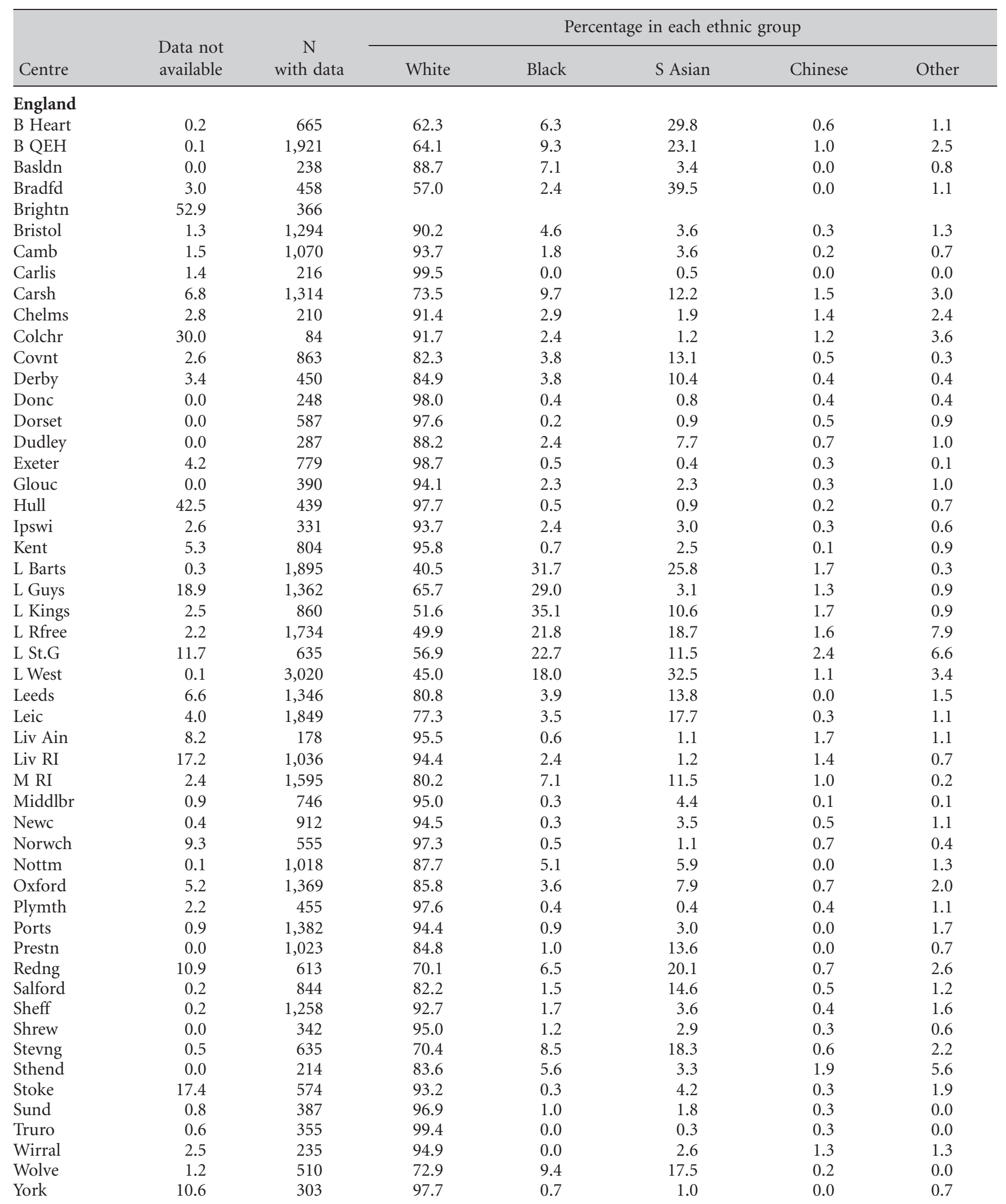


Table 2.10. Continued

\begin{tabular}{|c|c|c|c|c|c|c|c|}
\hline \multirow[b]{2}{*}{ Centre } & \multirow{2}{*}{$\begin{array}{l}\text { Data not } \\
\text { available }\end{array}$} & \multirow{2}{*}{$\begin{array}{c}\mathrm{N} \\
\text { with data }\end{array}$} & \multicolumn{5}{|c|}{ Percentage in each ethnic group } \\
\hline & & & White & Black & S Asian & Chinese & Other \\
\hline \multicolumn{8}{|l|}{$\mathrm{N}$ Ireland } \\
\hline Antrim & 0.0 & 224 & 99.1 & 0.0 & 0.9 & 0.0 & 0.0 \\
\hline Belfast & 1.3 & 677 & 99.0 & 0.1 & 0.7 & 0.1 & 0.0 \\
\hline Newry & 0.0 & 191 & 99.5 & 0.0 & 0.0 & 0.5 & 0.0 \\
\hline Ulster & 0.0 & 137 & 97.1 & 0.0 & 2.2 & 0.7 & 0.0 \\
\hline West NI & 0.0 & 272 & 98.9 & 0.4 & 0.4 & 0.4 & 0.0 \\
\hline \multicolumn{8}{|l|}{ Scotland } \\
\hline Abrdn & 57.4 & 204 & & & & & \\
\hline Airdrie & 67.4 & 112 & & & & & \\
\hline D \& Gall & 90.2 & 12 & & & & & \\
\hline Dundee & 53.5 & 186 & & & & & \\
\hline Dunfn & 80.9 & 53 & & & & & \\
\hline Edinb & 92.9 & 50 & & & & & \\
\hline Glasgw & 91.9 & 119 & & & & & \\
\hline Inverns & 8.9 & 204 & 99.0 & 0.0 & 1.0 & 0.0 & 0.0 \\
\hline Klmarnk & 55.0 & 135 & & & & & \\
\hline \multicolumn{8}{|l|}{ Wales } \\
\hline Bangor & 31.2 & 75 & 97.3 & 1.3 & 0.0 & 1.3 & 0.0 \\
\hline Cardff & 21.5 & 1,205 & 93.6 & 1.1 & 4.0 & 0.6 & 0.7 \\
\hline Clwyd & 22.8 & 105 & 99.0 & 0.0 & 0.0 & 1.0 & 0.0 \\
\hline Swanse & 1.2 & 651 & 98.3 & 0.3 & 1.2 & 0.0 & 0.2 \\
\hline Wrexm & 0.0 & 237 & 99.2 & 0.4 & 0.4 & 0.0 & 0.0 \\
\hline England & 5.4 & 42,254 & 77.4 & 8.3 & 11.9 & 0.7 & 1.7 \\
\hline $\mathrm{N}$ Ireland & 0.6 & 1,501 & 98.9 & 0.1 & 0.7 & 0.3 & 0.0 \\
\hline Scotland & 75.1 & 1,075 & & & & & \\
\hline Wales & 15.1 & 2,273 & 95.9 & 0.7 & 2.5 & 0.4 & 0.4 \\
\hline UK & 11.4 & 47,103 & 79.4 & 7.5 & 10.8 & 0.7 & 1.5 \\
\hline
\end{tabular}

Percentage breakdown is not shown for centres with less than $50 \%$ data completeness, but these centres are included in national averages Blank cells - less than $50 \%$ data completeness

Appendix $\mathrm{H}$ ethnicity coding

$2,007 \mathrm{pmp}$ in 2010) in the age-group 75-79 years before showing a reducing prevalence rate in age groups over 80 years. Crude prevalence rates in males exceeded those of females for all age groups, peaking in age group 75-79 years at 2,918 pmp and for females in age group 65-69 years at 1,460 pmp. Survival of males and females on RRT is described in chapter 5 .

\section{Ethnicity}

Fifty-one of the 71 centres $(71.8 \%)$ provided ethnicity data that were at least $90 \%$ complete (table 2.10 ) and this was an improvement compared with 49 of $72(68.1 \%)$ in 2010 and with 36 centres in 2006. Ethnicity completeness for prevalent RRT patients improved in the UK from $87.4 \%$ in 2010 to $88.6 \%$ in 2011 with $94.6 \%$ ethnicity completeness in England in 2011 and $99.4 \%$ in Northern Ireland. Completeness of ethnicity data was highest in prevalent transplant patients. This may relate to the fact that the intensive work-up for transplantation may increase the recording of data.

In 2011, 20.6\% of the prevalent UK RRT population (with ethnicity assigned) were from ethnic minorities (22.6\% in England). The proportion of the prevalent UK RRT population (with ethnicity assigned) from ethnic minorities in Wales, Scotland and Northern Ireland were very small, although it should be noted that there was a high level of missing ethnicity data in Scotland. The ONS estimates that approximately 12\% of the UK general population are designated as belonging to an ethnic minority [1]. The number of patients reported to the UKRR as receiving RRT and belonging to an ethnic minority has doubled in the last 5 years which may be due to improvements in coding of ethnicity data as well as an increasing incidence of ERF and increased referral rates in these populations.

Amongst the centres with more than 50\% returns there was wide variation in the proportion of patients 
Table 2.11. Primary renal diagnosis in prevalent RRT patients by age and gender on $31 / 12 / 2011$

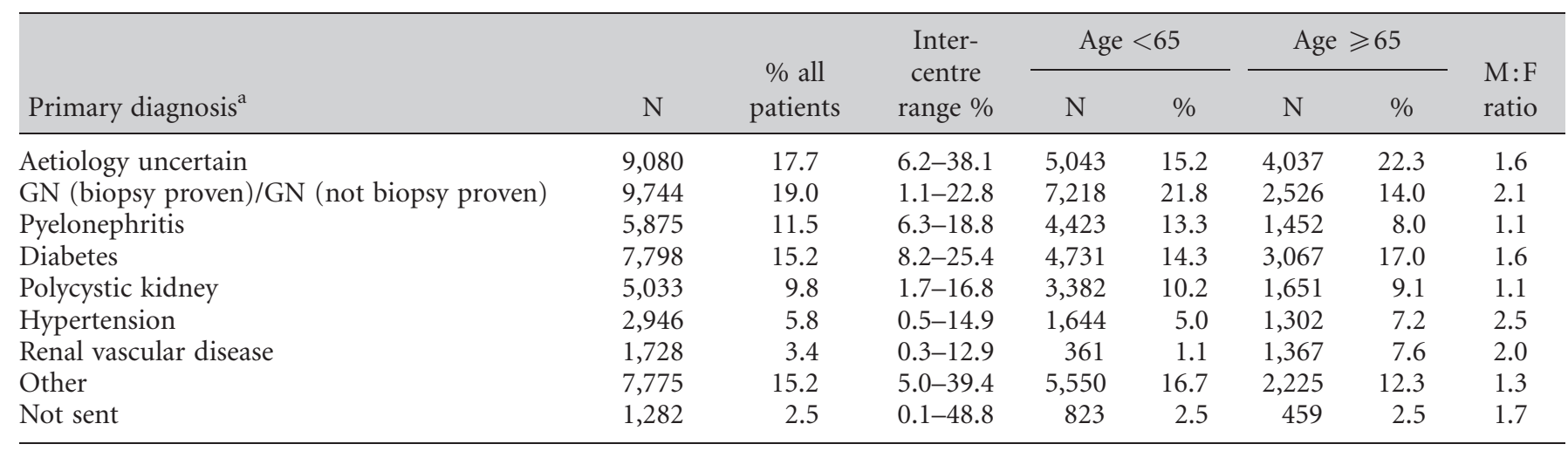

${ }^{a}$ Appendix H: ERA-EDTA coding

$\mathrm{GN}=$ glomerulonephritis

Excluded centres: $\geqslant 40 \%$ primary renal diagnosis aetiology uncertain (Colchr), $\geqslant 50 \%$ primary renal diagnosis not sent (L RFree)

from ethnic minorities, ranging from $0.5 \%$ in one centre (Carlisle) to over $50 \%$ in 3 centres: London Barts (59.5\%), London West (55.0\%) and London Royal Free (50.1\%). Three additional centres had over $40 \%$ of prevalent patients from ethnic minorities: Bradford (43.0\%), London Kings (48.4\%) and London St Georges (43.1\%). Thirteen of twenty-three (56.5\%) transplanting centres had an ethnic minority population greater than $10 \%$ compared with $27.1 \%$ (13/48) of non-transplanting centres.

Ethnicity also impacted the median age of the prevalent cohort. Those centres with an ethnic minority population of $>10 \%$ had a slightly lower median age (57 years vs. 59 years).

\section{Primary renal diagnosis}

Data for primary renal diagnosis (PRD) were not complete for $2.5 \%$ of patients and there remained a marked inter-centre difference in completeness of data returns (table 2.11). London Royal Free was excluded from the following analyses as it had $\geqslant 50 \%$ primary renal diagnosis data missing. The UKRR remains concerned that some centres have very high rates of primary renal diagnosis coded as 'uncertain' (EDTA codes 00 and 10). It is accepted that inevitably there will be a number of patients with uncertain aetiology and that the proportion of these patients will vary as the definitions of renal vascular disease, hypertensive nephropathy and chronic glomerulonephritis (GN) without tissue diagnosis remain relatively subjective. However, some centres with very high rates of 'uncertain' as the primary renal diagnosis appear to also have fewer patients with the more objective diagnoses such as polycystic kidney disease or biopsy-proven GN. It is believed that the software in these centres defaults any missing data to 'uncertain' (EDTA code 00).

One centre with $\geqslant 40 \%$ 'uncertain' primary renal diagnosis (Colchester, 47\%) has been excluded from the inter-centre analysis and the UK and national totals have been adjusted. These centres with either a high proportion of primary renal diagnosis 'uncertain' or a high proportion of missing data have also been excluded from other analyses where PRD is included in the casemix adjustment. There was wide inter-centre variation in the proportion of primary renal diagnoses in the RRT prevalent population not submitted, but this is improving in most centres. There were 4 centres with $>15 \%$ not sent (Wirral 27.0\%, Brighton 22.7\%, Salford $17.4 \%$, London Royal Free 50.2\%). Uncertain primary renal diagnosis also ranged widely between centres and 3 centres had $>30 \%$ uncertain diagnosis (Ipswich 30.6\%, Liverpool RI 37.2\%, Colchester 46.7\%).

Glomerulonephritis remained the most common primary renal diagnosis in the 2011 prevalent cohort at $19.0 \%$ (table 2.11). The change in coding in this year's analysis from glomerulonephritis that is biopsy proven to including those that are not biopsy proven is reflected in an increase in prevalence from 16\% in 2010 to $19 \%$ in 2011. Diabetes accounted for $15.2 \%$ of renal disease in the prevalent patients on RRT, although it was more common in the $\geqslant 65$ year age group compared to the younger group (17.0\% vs. $14.3 \%)$. This contrasted with the pattern seen in incident patients where diabetes was the predominant specific diagnostic code in $25.0 \%$ of new RRT patients. This reflects the different ages and survival of patients with these diagnoses; it is the younger fitter patients who survive longest and contribute highly to the prevalent numbers. Younger 
Table 2.12. Transplant: dialysis ratios by age and primary renal disease in the prevalent RRT population on 31/12/2011

\begin{tabular}{lcc}
\hline & \multicolumn{2}{c}{ Transplant: dialysis ratio } \\
\cline { 2 - 3 } Primary diagnosis $^{\mathrm{a}}$ & $<65$ & $\geqslant 65$ \\
\hline Aetiology uncertain & 1.8 & 0.3 \\
GN (biopsy proven)/GN (not biopsy & 2.2 & 0.7 \\
proven) & & \\
Pyelonephritis & 2.4 & 0.4 \\
Diabetes & 0.8 & 0.1 \\
Polycystic kidney & 2.2 & 1.3 \\
Hypertension & 1.1 & 0.3 \\
Renal vascular disease & 0.9 & 0.1 \\
Other & 1.8 & 0.3 \\
Not sent & 1.4 & 0.2 \\
\hline
\end{tabular}

${ }^{a}$ Appendix H ERA-EDTA coding

$\mathrm{GN}=$ glomerulonephritis

Excluded centres: $\geqslant 40 \%$ primary renal diagnosis aetiology uncertain (Colchr), $\geqslant 50 \%$ primary renal diagnosis not sent (L RFree)

patients (age $<65$ years) are more likely to have GN or pyelonephritis and less likely to have renal vascular disease or hypertension as the cause of their renal failure.

The male:female ratio was greater than unity for all primary renal diagnoses (table 2.11). The gender imbalance may be influenced by the presence of factors such as hypertension, atheroma and renal vascular disease, which are more common in males, more common with increasing age and which may increase the rate of progression of kidney disease.

In individuals aged less than 65 years, renal transplantation to dialysis ratio was greater than 1 in all PRD groups except diabetes and renovascular disease. In those aged $>65$ years, dialysis was more prevalent than renal transplantation in all PRD groups except PKD (table 2.12).

\section{Diabetes}

Diabetes included all prevalent patients with type 1 or type 2 diabetes as primary renal diagnosis (ERA-EDTA coding) and did not include patients with diabetes as a comorbidity. This analysis did not differentiate between type 1 and type 2 diabetes as this distinction was not made in the data submitted by most centres.

The number of prevalent patients with diabetes as a primary renal diagnosis increased $7 \%$ to 7,798 in 2011 , from 7,282 in 2010 , representing $14.7 \%$ of all prevalent patients (compared with $13.5 \%$ in 2006) (table 2.13). The median age at start of RRT for patients with diabetes (56 years) was 9 years higher compared with patients without diabetes ( 47 years), although the median age at the end of 2011 for prevalent diabetic patients was only 3.5 years higher than for individuals without diabetes. This reflects
Table 2.13. Age relationships in diabetic and non-diabetic patients and modality in prevalent RRT patients on 31/12/2011

\begin{tabular}{lcc}
\hline & $\begin{array}{c}\text { Diabetic } \\
\text { patients }\end{array}$ & $\begin{array}{c}\text { Non-diabetic } \\
\text { patients }\end{array}$ \\
\hline Number & 7,798 & 42,181 \\
M:F ratio & 1.60 & 1.54 \\
Median age on 31/12/2011 & 61 & 58 \\
Median age at start of RRT & 56 & 47 \\
Median years on RRT & 3.4 & 6.5 \\
\% HD & 61 & 40 \\
\% PD & 9 & 7 \\
\% transplant & 30 & 53 \\
\hline
\end{tabular}

Excluded centres: $\geqslant 40 \%$ primary renal diagnosis aetiology uncertain (Colchr), $\geqslant 50 \%$ primary renal diagnosis not sent (L RFree)

Diabetic patients: patients with a primary renal disease code of diabetes

Non-diabetic patients: all patients excluding diabetic patients and patients with a missing primary renal disease code

Median age at start of RRT was calculated from the most recent RRT start date

Patients with an initial treatment modality of transferred in or transferred out were excluded from the calculation of median age at start of RRT and median years on RRT, since their treatment start date was not accurately known

Patients without a treatment modality code were excluded from calculating the $\%$ per treatment modality

reduced survival for patients with diabetes compared with patients without diabetes on RRT. Median time on RRT for patients with diabetes was less when compared with patients without diabetes (3.4 years vs. 6.5 years) and this difference in survival between diabetic patients and non-diabetic patients has not changed over the last 5 years. Patients with diabetes starting RRT in Scotland were 3 years younger and in Northern Ireland 3 years older compared with the UK average age of patients with diabetes starting RRT (data not shown).

Diabetes as the primary renal diagnosis also influenced the modality distribution. The predominant mode of treatment for patients with diabetes was HD (61\%) compared with $40 \%$ in individuals who had a different primary renal diagnosis (table 2.13). The percentage of patients with a functioning transplant was much lower in prevalent patients with diabetes than in prevalent patients without diabetes ( $30 \%$ vs. 53\%). However, the proportion of patients with diabetes as PRD with a functioning transplant has increased since 2004 when only $26 \%$ of patients with diabetes had a functioning transplant. For older patients with diabetes (age $\geqslant 65$ years), 9.0\% had a functioning transplant compared with $28.8 \%$ of their peers without diabetes (table 2.14). In Northern Ireland, $23.4 \%$ of prevalent patients with diabetes had a functioning transplant compared with the UK average of $30.2 \%$ 
Table 2.14. Treatment modalities by age and diabetes status in UK countries on $31 / 12 / 2011$

\begin{tabular}{lccccc}
\hline & \multicolumn{2}{c}{$<65$ years } & & \multicolumn{2}{c}{$\geqslant 65$ years } \\
\cline { 2 - 3 } \cline { 5 - 6 } & Diabetes & $\begin{array}{c}\text { Non- } \\
\text { diabetic }\end{array}$ & & Diabetes & $\begin{array}{c}\text { Non- } \\
\text { diabetic }\end{array}$ \\
\hline Number & 4,731 & 27,621 & & 3,067 & 14,560 \\
\% HD & 47.9 & 28.4 & & 81.1 & 62.2 \\
\% PD & 8.1 & 5.7 & & 9.9 & 9.0 \\
\% transplant & 44.0 & 65.9 & & 9.0 & 28.8 \\
\hline
\end{tabular}

Excluded centres: $\geqslant 40 \%$ primary renal diagnosis aetiology uncertain (Colchr), $\geqslant 50 \%$ primary renal diagnosis not sent (L RFree)

Diabetic patients: patients with a primary renal disease code of diabetes

Non-diabetic patients: all patients excluding diabetic patients and patients with a missing primary renal disease code

Excludes all patients without a treatment modality code

although on average the Northern Ireland patients with diabetes were older by three years (data not shown). A higher proportion of prevalent patients without diabetes (18.0\%) were on home dialysis therapies (home HD and PD) compared with prevalent patients with diabetes $(14.0 \%)$.

\section{Modalities of treatment}

Transplantation was the most common treatment modality (48.6\%) for prevalent RRT patients in 2011, followed closely by centre-based HD (42.4\%) in either hospital centre $(21.8 \%)$ or satellite unit (20.6\%) (figure 2.6). Home therapies made up the remaining $9.1 \%$ of treatment therapies, largely PD in its different formats $(7.6 \%)$ which was similar to 2010 . The proportion on continuous ambulatory peritoneal dialysis (CAPD) and automated PD (APD) was 3.9\% and 3.7\% respectively, though the proportion on APD may be an under-estimate due to centre level coding issues which mean the UKRR cannot always distinguish between these therapies. The term CAPD has been used for patients receiving non-disconnect as well as disconnect

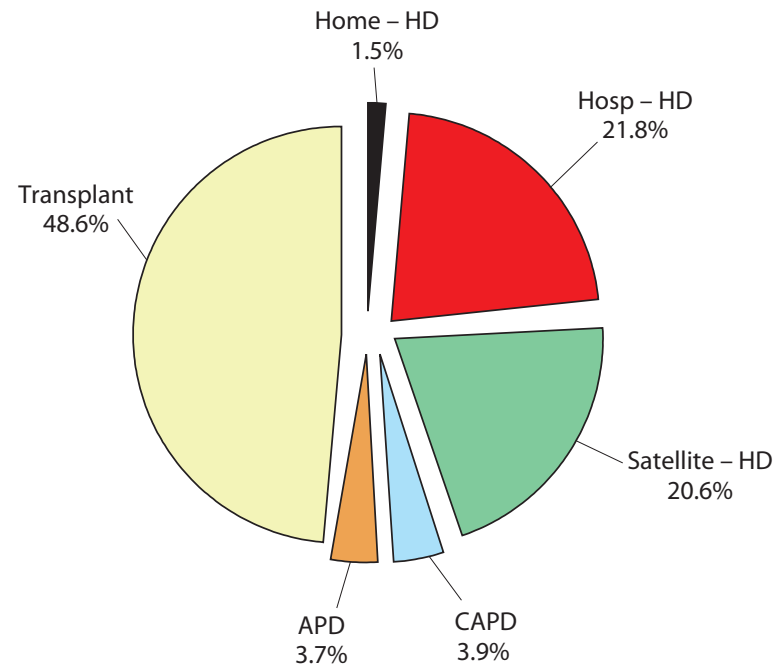

Fig. 2.6. Treatment modality in prevalent RRT patients on $31 / 12 / 2011$

CAPD systems, because the proportion of patients using non-disconnect systems was very small.

As mentioned earlier, treatment modality was related to patient age. Younger patients (age $<65$ years), were more likely to have a functioning transplant $(62.7 \%)$ when compared with patients aged over 65 years (25.0\%) (table 2.15). HD was the principal modality in the older patients $(65.7 \%)$. In the elderly using the proportion of renal replacement therapy patients transplanted can be misleading as this depends on approaches to dialysis and conservative care in this age group.

Figure 2.7 shows the association between age and RRT modality. Beyond 54 years of age, transplant prevalence declined, whilst HD prevalence increased. The proportion of each age group treated by $\mathrm{PD}$ remained more stable across the age spectrum.

The proportion of prevalent dialysis patients receiving $\mathrm{HD}$, ranged from $64.9 \%$ in Derby to $100 \%$ in Colchester (table 2.16).

Table 2.15. Percentage of prevalent RRT patients by dialysis and transplant modality by centre on $31 / 12 / 2011$

\begin{tabular}{|c|c|c|c|c|c|c|c|c|}
\hline \multirow[b]{2}{*}{ Country } & \multicolumn{4}{|c|}{$<65$ years } & \multicolumn{4}{|c|}{$\geqslant 65$ years } \\
\hline & $\mathrm{N}$ & $\% \mathrm{HD}$ & $\% \mathrm{PD}$ & $\%$ transplant & $\mathrm{N}$ & $\% \mathrm{HD}$ & $\% \mathrm{PD}$ & $\%$ transplant \\
\hline N Ireland & 941 & 32.7 & 4.8 & 62.5 & 569 & 73.3 & 5.8 & 20.9 \\
\hline Scotland & 2,952 & 32.6 & 4.9 & 62.6 & 1,372 & 66.8 & 7.7 & 25.5 \\
\hline Wales & 1,675 & 27.0 & 6.4 & 66.5 & 1,002 & 63.8 & 10.1 & 26.1 \\
\hline
\end{tabular}

All patients without a treatment modality code were excluded 


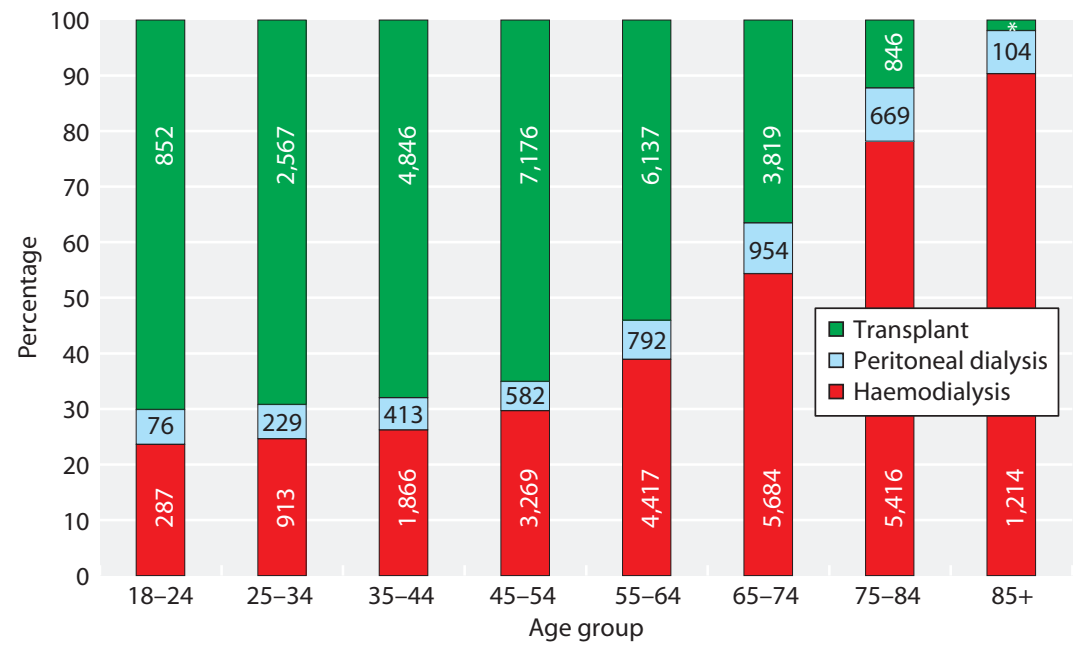

Fig. 2.7. Treatment modality distribution by age in prevalent RRT patients on $31 / 12 / 2011$
Overall, the proportion of dialysis patients treated in a satellite haemodialysis unit has increased to $41.5 \%$ this year compared to $39.9 \%$ in 2010 , and $36 \%$ in 2009 . Although there are satellite units in Scotland, the data provided for 2011 did not distinguish between main centre and satellite unit haemodialysis. In 2011, the number of centres that had more than $50 \%$ of their haemodialysis activity taking place in satellite units was 25, a slight decline from 2010 (table 2.16 and figure 2.8). There was also wide variation between centres in the proportion of dialysis patients on APD treatment, ranging from $0 \%$ to $21.4 \%$ (table 2.16 ). Ten of the 70 centres with a PD programme had no patients on APD, whilst in four Northern Ireland centres almost all PD patients were on this form of the modality.

\section{Home haemodialysis}

The use of home HD as a RRT peaked in 1982 when almost 2,200 patients were estimated to be on this therapy, representing $61 \%$ of HD patients reported to the ERA-EDTA registry at that time. The fall in the use of this modality to just 445 patients $(2.4 \%$ of $\mathrm{HD}$ patients) in 2006 was probably due to an increase in availability and uptake of renal transplantation, and also the similar expansion of hospital HD provision with the introduction of satellite units. In the last seven years there has been renewed interest in home HD and a target of $15 \%$ of HD patients on this modality has been suggested [5]. Equipment changes and patient choice has helped drive this change. Since 2006 there has been a gradual increase in the proportion of prevalent patients receiving haemodialysis in their own homes so that in 2011 it reached $3.9 \%$ of HD patients $(\mathrm{n}=905$, figure 2.2 and table 2.16). These numbers may be an under-estimate as some centres have been unable to submit data for patients coded as home HD and work is on-going to address this.

In 2011, the percentage of dialysis patients receiving home HD varied from $0 \%$ in 9 centres, to greater than $5 \%$ in 16 centres (table 2.16). In the UK, the overall prevalence of home haemodialysis has increased from $2.9 \%$ in 2010 to $3.4 \%$ in 2011 .

The proportion of patients receiving home haemodialysis was greatest in Wales at 5.9\%, compared with $3.7 \%$ in N.Ireland, $3.3 \%$ in England and $2.6 \%$ in Scotland (figure 2.8, table 2.16). These proportions are similar to 2010. Forty-three renal centres in England had an increase in the proportion of individuals on home haemodialysis compared with 2010.

In 2007, for comparison, the proportion of patients receiving home haemodialysis was $2 \%$ in each of the four UK countries.

\section{Change in modality}

The relative proportion of RRT modalities in prevalent patients has changed dramatically over the past decade. The main features are depicted in figure 2.9, which describes a sustained decrease in the proportion of patients treated by PD after 2000. Possible explanations for this change include recently published evidence indicating that the equivalent survival demonstrated between $\mathrm{HD}$ and PD was only maintained for the first 2-3 years [6] and recent concerns regarding the risk of encapsulating peritoneal sclerosis which might result in patients being switched from PD to HD after a fixed time interval. Analysis of UKRR data has not supported this explanation however as the vintage of PD patients has not changed substantially over the last 8 years. The 
Table 2.16. Percentage of prevalent dialysis patients by dialysis modality by centre on $31 / 12 / 2011$

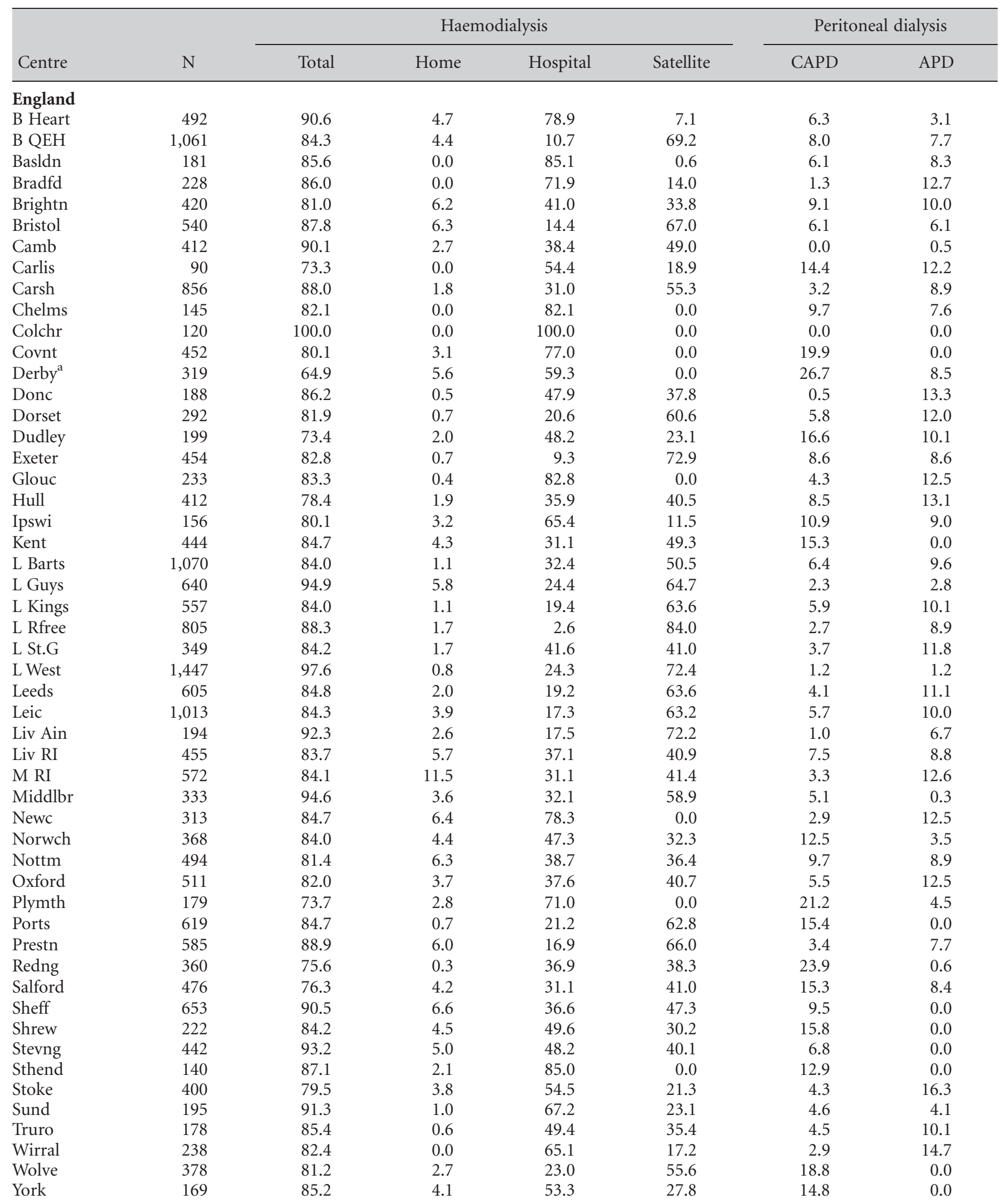


Table 2.16. Continued

\begin{tabular}{|c|c|c|c|c|c|c|c|}
\hline \multirow[b]{2}{*}{ Centre } & \multirow[b]{2}{*}{$\mathrm{N}$} & \multicolumn{4}{|c|}{ Haemodialysis } & \multicolumn{2}{|c|}{ Peritoneal dialysis } \\
\hline & & Total & Home & Hospital & Satellite & CAPD & APD \\
\hline \multicolumn{8}{|l|}{ N Ireland ${ }^{\mathrm{b}}$} \\
\hline Antrim & 146 & 90.4 & 2.7 & 87.7 & 0.0 & 2.7 & 6.9 \\
\hline Belfast & 258 & 88.4 & 5.8 & 82.6 & 0.0 & 1.2 & 10.1 \\
\hline Newry & 123 & 90.2 & 2.4 & 87.8 & 0.0 & 0.0 & 9.8 \\
\hline Ulster & 108 & 97.2 & 3.7 & 93.5 & 0.0 & 0.0 & 2.8 \\
\hline West NI & 168 & 88.7 & 2.4 & 86.3 & 0.0 & 0.6 & 10.7 \\
\hline \multicolumn{8}{|l|}{ Scotland ${ }^{c}$} \\
\hline Abrdn & 237 & 90.3 & 1.7 & 88.6 & 0.0 & 4.6 & 5.1 \\
\hline Airdrie & 183 & 94.5 & 0.0 & 94.5 & 0.0 & 1.6 & 3.8 \\
\hline D \& Gall & 63 & 77.8 & 1.6 & 76.2 & 0.0 & 6.4 & 15.9 \\
\hline Dundee & 205 & 89.3 & 0.0 & 89.3 & 0.0 & 2.0 & 8.8 \\
\hline Dunfn & 174 & 83.9 & 0.0 & 83.9 & 0.0 & 0.0 & 16.1 \\
\hline Edinb & 301 & 86.7 & 2.0 & 84.7 & 0.0 & 4.0 & 9.3 \\
\hline Glasgw & 671 & 92.7 & 4.9 & 87.8 & 0.0 & 2.1 & 5.2 \\
\hline Inverns & 101 & 82.2 & 5.0 & 77.2 & 0.0 & 6.9 & 10.9 \\
\hline Klmarnk & 192 & 76.6 & 3.7 & 72.9 & 0.0 & 2.1 & 21.4 \\
\hline \multicolumn{8}{|l|}{ Wales } \\
\hline Bangor & 109 & 80.7 & 11.9 & 52.3 & 16.5 & 5.5 & 13.8 \\
\hline Cardff & 597 & 82.9 & 5.4 & 16.8 & 60.8 & 12.4 & 4.7 \\
\hline Clwyd $^{\mathrm{d}}$ & 71 & 88.7 & 5.6 & 83.1 & 0.0 & 9.9 & 1.4 \\
\hline Swanse & 416 & 86.1 & 6.5 & 50.0 & 29.6 & 10.6 & 3.4 \\
\hline Wrexm & 108 & 81.5 & 0.9 & 75.9 & 4.6 & 18.5 & 0.0 \\
\hline England & 22,654 & 85.5 & 3.3 & 35.2 & 47.0 & 7.5 & 6.8 \\
\hline N Ireland ${ }^{\mathrm{b}}$ & 803 & 90.3 & 3.7 & 86.6 & 0.0 & 1.0 & 8.6 \\
\hline Scotland ${ }^{c}$ & 2,127 & 88.3 & 2.6 & 85.7 & 0.0 & 2.8 & 8.9 \\
\hline Wales & 1,301 & 83.9 & 5.9 & 38.9 & 39.1 & 11.6 & 4.5 \\
\hline UK & 26,885 & 85.8 & 3.4 & 40.9 & 41.5 & 7.1 & 6.9 \\
\hline
\end{tabular}

${ }^{a}$ In 2010 it was reported that Derby had a home haemodialysis prevalence of $14.3 \%$. This was inaccurate due to a data error. The actual prevalence was $2.8 \%$

${ }^{\mathrm{b}}$ There are no satellite centres in Northern Ireland

${ }^{\mathrm{c}}$ All haemodialysis patients in Scotland are shown as receiving treatment at home or in centre as no data is available regarding satellite dialysis

${ }^{\mathrm{d}} 13 \mathrm{HD}$ and $12 \mathrm{PD}$ patients from Clwyd were not included in this analysis

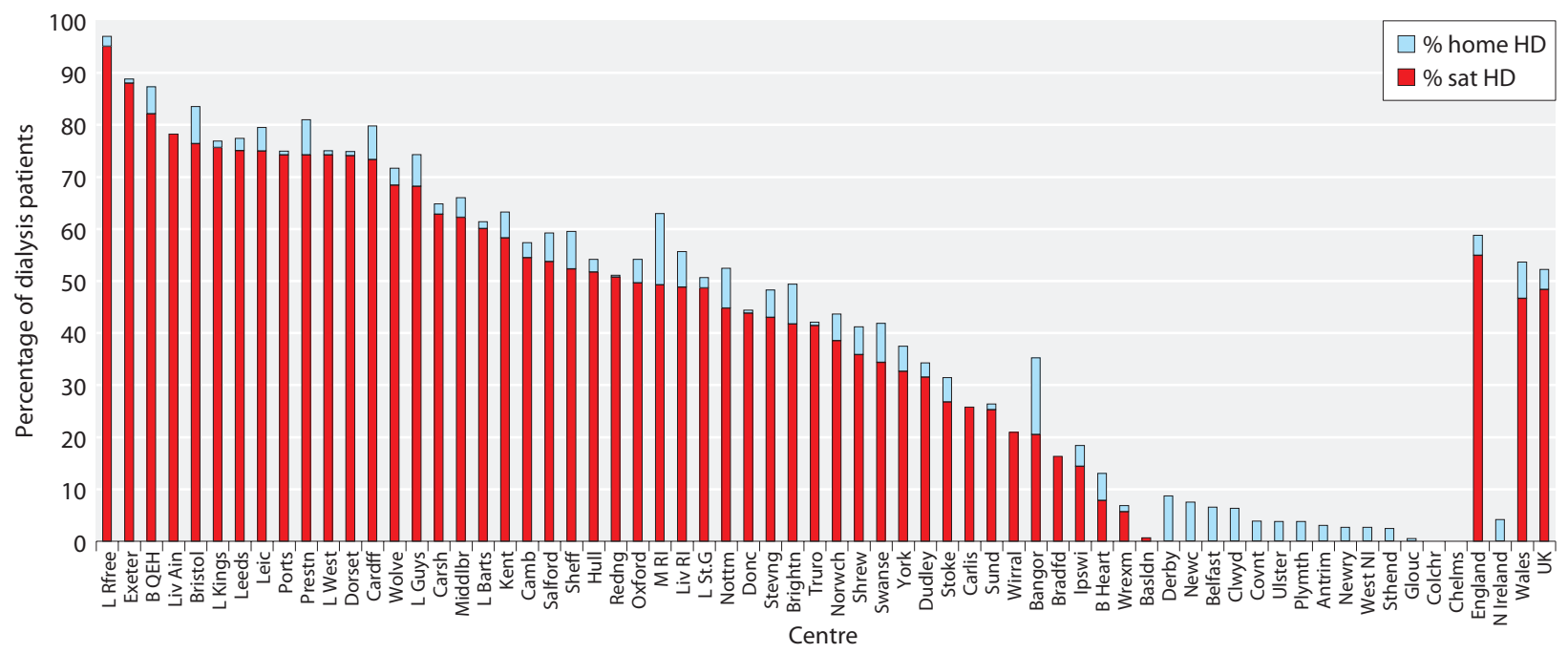

Fig. 2.8. Percentage of prevalent haemodialysis patients treated with satellite or home haemodialysis by centre on $31 / 12 / 2011$ ${ }^{*}$ Scottish centres excluded as information on satellite HD was not available. No centres in Northern Ireland have satellite dialysis units 


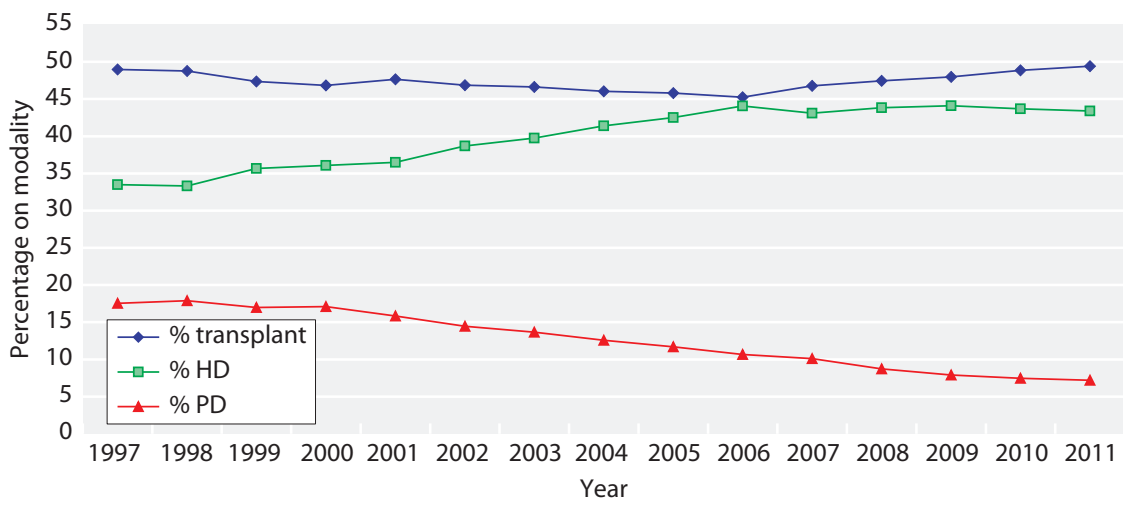

Fig. 2.9. Modality changes in prevalent RRT patients from 1997-2011 reduction in prevalent $\mathrm{PD}$ patients can be explained due to a decrease in the number of new patients who were started on peritoneal dialysis in 2010 and 2011 and also to the declining proportion of patients starting RRT on peritoneal dialysis since 2001. The determinants of this pattern may be multi-factorial and include: an increase in HD capacity with the proliferation of satellite units (figure 2.10), the effect of patient or physician choice regarding the treatment modality at start of RRT, the general health and fitness of patients starting RRT, some of whom may be deemed less capable of undertaking PD independently, and the rise in the number of patients receiving a live related transplant who may otherwise have gone onto PD. With the advent of assisted PD (more commonly used in France) [7] in conjunction with the increasing age of PD patients, there may be potential for some reversal or slowing in this decline. The proposed introduction of dialysis tariffs in England may well result in further changes to the types of treatment patients receive in England.
The proportion of patients treated with HD has plateaued in the last three years. The proportion of patients with a functioning transplant had been on a slight downward trend but this has reversed since 2007, probably due to continued increases in living organ and non-heart beating donation [8].

Figure 2.10 depicts in more detail the modality changes in the prevalent dialysis population during this time and highlights a sustained reduction in the proportion of patients treated by CAPD. There was a sustained increase in the proportion of prevalent HD patients treated at satellite units with a steady decline in hospital centre haemodialysis since 2004 .

\section{International comparisons}

For international comparisons 2010 prevalence rates are given as 2011 data were not available from the other

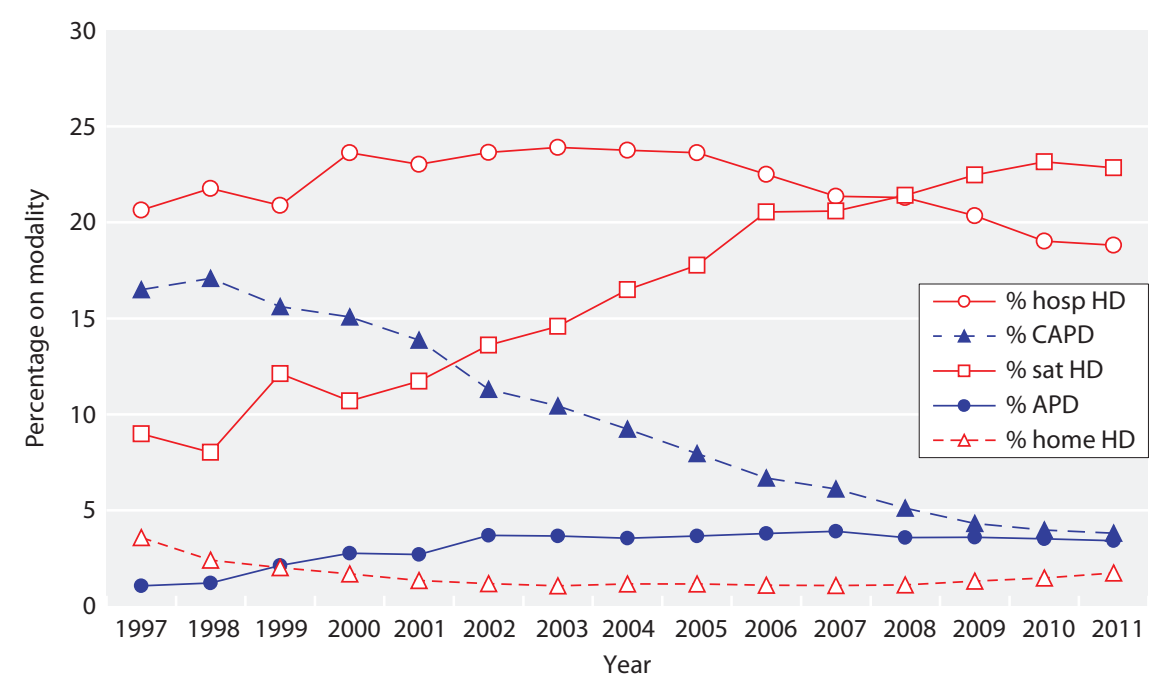

Fig. 2.10. Detailed dialysis modality changes in prevalent RRT patients from 1997-2011 * Scottish centres excluded as information on satellite HD was not available 


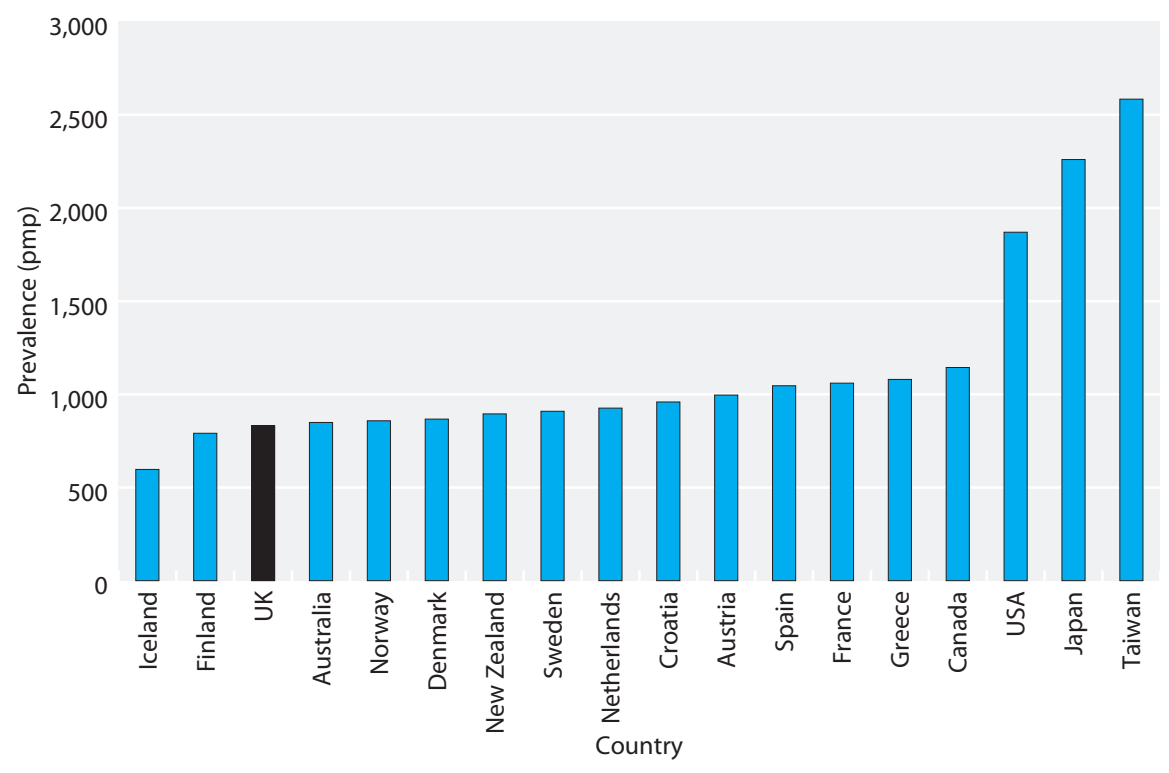

Fig. 2.11. RRT prevalence rates (pmp) by country in 2010

Non UK data from USRDS countries. Prevalence rates in the UK were similar to those in most other Northern European countries but lower than in Southern Europe and Belgium and far lower than in the USA (figure 2.11). This will in part reflect differences in incidence rates and also conservative management practices between countries in addition to other individual level and health care system differences.

\section{Summary}

There continues to be growth across the UK in prevalent patients on RRT with regional and centre level variation. There was no real difference in prevalence rates between the four nations of the UK once adjusted for background population characteristics. In general, areas with large ethnic minority populations had higher standardised prevalence ratios. There were increasing numbers of patients on $\mathrm{HD}$ and those with a functioning transplant and falling numbers on PD. The prevalence rate in the over 80 year olds has doubled since 2005. There have been substantial increases in home HD use in some areas although several centres are still unable to offer this modality.

Conflicts of interest: none

\section{References}

1 Office for National Statistics. www.statistics.gov.uk

2 Office of the national statistics. The classification of ethnic groups. (www.statistics.gov.uk)

3 Byrne C, Steenkamp R, Castledine C, Ansell D, Feehally J. UK Renal Registry report 2008. UK Renal Registry Bristol; Chapter 4: p 41-67

4 Ansell D, Feest T: The sixth annual report. Chapter 17: Social deprivation on renal replacement therapy. Bristol, UK Renal Registry, 2003

5 NICE 2002. Technology appraisal No 48. National Institute Clinical Excellence. www.nice.org.uk
6 McDonald SP, Marshall MR, Johnson DW, Polkinghorne KR. Relationship between Dialysis Modality and Mortality. J Am Soc Nephrol. 2009; 20(1):155-63

7 Couchoud C, Stengel B, Landais P, Aldigier J-C, de Cornelissen F, Dabot $\mathrm{C}$, et al.. The renal epidemiology and information network (REIN): a new registry for end-stage renal disease in France. Nephrol Dial Transplant. 2006; 21(2):411-8

8 NHS Blood and Transplant activity report 2009/2010. Transplant activity in the UK.http://www.organdonation.nhs.uk/ukt/statistics/transplant_ activity_report/current_activity_reports/ukt/activity_report_2009_10.pdf 